\title{
Heteroleptic Cyclometalated Iridium(III) Complexes Displaying Blue Phosphorescence in Solution and Solid State at Room Temperature
}

\author{
Cheng-Han Yang, Shih-Wen Li, and Yun $\mathrm{Chi}^{*}$ \\ Department of Chemistry, National Tsing Hua University, Hsinchu 300, Taiwan \\ Yi-Ming Cheng, Yu-Shan Yeh, Pi-Tai Chou, ${ }^{*}$ and Gene-Hsiang Lee \\ Department of Chemistry and Instrumentation Center, National Taiwan University, \\ Taipei 106, Taiwan \\ Chih-Hsiang Wang and Ching-Fong Shu \\ Department of Applied Chemistry, National Chiao Tung University, Hsinchu 300, Taiwan \\ Received March 1, 2005
}

\begin{abstract}
A series of heteroleptic $\operatorname{lr}($ III) metal complexes 1-3 bearing two $\mathrm{N}$-phenyl-substituted pyrazoles and one 2-pyridyl pyrazole (or triazole) ligands were synthesized and characterized to attain highly efficient, room-temperature blue phosphorescence. The $\mathrm{N}$-phenylpyrazole ligands, dfpz $\mathrm{H}=1$-(2,4-difluorophenyl)pyrazole, $\mathrm{fpz} \mathrm{H}=1$-(4-fluorophenyl)pyrazole, dfmpzH = 1-(2,4-difluorophenyl)-3,5-dimethylpyrazole, and fmpzH = 1-(4-fluorophenyl)-3,5-dimethylpyrazole, show a similar reaction pattern with respect to the typical cyclometalated $\left(C^{\wedge} N\right)$ chelate, which utilizes its orthosubstituted phenyl segment to link with the central $\operatorname{Ir}(\mathrm{III})$ atom, while the second 2-pyridylpyrazole (or triazole) ligand, namely, fppzH = 3-(trifluoromethyl)-5-(2-pyridyl)pyrazole, fptzH = 3-(trifluoromethyl)-5-(2-pyridyl)triazole, and $\mathrm{hptz} \mathrm{H}=3$-(heptafluoropropyl)-5-(2-pyridyl)triazole, undergoes typical anionic $\left(\mathrm{N}^{\wedge} \mathrm{N}\right)$ chelation to complete the octahedral framework. X-ray structural analyses on complexes [(dfpz $\left.)_{2} \operatorname{Ir}(\mathrm{fppz})\right](\mathbf{1 a})$ and [(fmpz $\left.)_{2} \operatorname{lr}(\mathrm{hptz})\right]$ (3d) were established to confirm their molecular structures. Increases of the $\pi \pi^{*}$ energy gaps of the $\operatorname{Ir}($ III) metal complexes were systematically achieved with two tuning strategies. One involves the substitution for one or two fluorine atoms at the $\mathrm{N}$-phenyl segment or the introduction of two electron-releasing methyl substituents at the pyrazole segment of the $\mathrm{H}\left(\mathrm{C}^{\wedge} \mathrm{N}\right)$ ligands. Alternatively, we have applied the more electron-accepting triazolate in place of the pyrazolate segment for the third $\left(\mathrm{N}^{\wedge} \mathrm{N}\right) \mathrm{H}$ ligand. Our results, on the basis of steady-state, relaxation dynamics, and theoretical approaches, lead to a conclusion that, for complexes 1-3, the weakening of iridium metal-ligand bonding strength in the $T_{1}$ state plays a crucial role for the fast radiationless deactivation. For the case of [(fmpz) 21 (hptz)] (3d), a thermal deactivation barrier of $4.8 \mathrm{kcal} / \mathrm{mol}$ was further deduced via temperature-dependent studies. The results provide a theoretical basis for future design and synthesis of the corresponding analogues suited to blue phosphorescent emitters.
\end{abstract}

One of the most important research subjects in the development of organic light-emitting diodes (OLEDs) is the development of phosphorescent metal complexes showing all three primary colors for full-color displays. Among phosphorescent complexes, green-emitting complexes have been known for years and have been successfully fabricated as green OLEDs with $\sim 100 \%$ internal quantum efficiency. ${ }^{1}$

* To whom correspondence should be addressed. E-mail: ychi@ mx.nthu.edu.tw (Y.C.).

7770 Inorganic Chemistry, Vol. 44, No. 22, 2005
Recently, red-emitting complexes have become accessible through selective incorporation of highly conjugated ancillary

(1) (a) Hua, F.; Kinayyigit, S.; Cable, J. R.; Castellano, F. N. Inorg. Chem. 2005, 44, 471. (b) Yang, C.-H.; Fang, K.-H.; Chen, C.-H.; Sun, I.-W. Chem. Commun. 2004, 2232. (c) Tokito, S.; Iijima, T.; Tsuzuki, T.; Sato, F. Appl. Phys. Lett. 2003, 83, 2459. (d) Lo, S.-C.; Namdas, E. B.; Burn, P. L.; Samuel, I. D. W. Macromolecules 2003, 36, 9721. (e) Bruce, D.: Richter, M. M. Anal. Chem. 2002, 74, 1340. (f) Adachi, C.; Baldo, M. A.; Forrest, S. R.; Thompson, M. E. Appl. Phys. Lett. 2000, 77, 904 .

10.1021/ic050311g CCC: $\$ 30.25$ C 2005 American Chemical Society Published on Web 09/20/2005 
groups into the ligand chromophore to extend the tripletstate lifetime ${ }^{2}$ as well as to circumvent their intrinsic obstacle, involving rapid nonradiative deactivation predicted by the energy gap law. ${ }^{3}$ Presently, researchers have turned their attention to the preparation and characterization of the remaining blue-emitting phosphorescent complexes. ${ }^{4}$ This task, however, is even more difficult to achieve than those of the other two cases. One major challenge lies in the selection of suitable chelate ligands that are able to form complexes with sufficiently large ligand-centered $\pi \pi^{*}$ transition energies and/ or metal-to-ligand charge transfer (MLCT) energies. Such an approach might inevitably raise the ligandcentered transition (or MLCT) to a region very close to the higher lying metal-centered dd states (or ligand field, LF, states) and then induce an efficient radiationless decay pathway through stretching the metal-ligand bonding. Thus, subtlety in the selection of metal elements as well as the ancillary ligands is indispensable so that the thermal deactivation of the metal centered dd states can be effectively suppressed to increase the yield of blue phosphorescence. ${ }^{5}$ To achieve this goal, a few attempts have been made through the use of strong field ancillary ligands such as $\mathrm{CO}$ or cyanide with an aim to increase the HOMO-LUMO electronic energy gaps and with the incorporation of third-row metal elements to strengthen metal-ligand bonding, thereby minimizing radiationless decay via energetically close-lying dd states. ${ }^{6}$ More recently, a series of blue-emitting Ir complexes $\left[(\operatorname{tpy})_{2} \operatorname{Ir}(\mathrm{LX})\right]$ (tpy $=2$-p-tolylpyridine; $\mathrm{LX}=$ acetylacetonato, bis(pyrazolyl)borate ligands and their analogues, diphosphine chelates, and tert-butyl isocyanide) have been reported. The results clearly demonstrate the feasibility

(2) (a) Tung, Y.-L.; Lee, S.-W.; Chi, Y.; Tao, Y.-T.; Chien, C.-H.; Cheng, Y.-M.; Chou, P.-T.; Peng, S.-M.; Liu, C.-S. J. Mater. Chem. 2005, 15, 460. (b) Kavitha, J.; Chang, S.-Y.; Chi, Y.; Yu, J.-K.; Hu, Y.-H.; Chou, P.-T.; Peng, S.-M.; Lee, G.-H.; Tao, Y.-T.; Chien, C.-H.; Carty, A. J. Adv. Funct. Mater. 2005, 15, 223. (c) Anthopoulos, T. D.; Frampton, M. J.; Namdas, E. B.; Burn, P. L.; Samuel, I. D. W. Adv. Mater. 2004, 16, 557. (d) Tsuboyama, A.; Iwawaki, H.; Furugori, M.; Mukaide, T.; Kamatani, J.; Igawa, S.; Moriyama, T.; Miura, S.; Takiguchi, T.; Okada, S.; Hoshino, M.; Ueno, K. J. Am. Chem. Soc. 2003, 125, 12971. (e) Adachi, C.; Baldo, M. A.; Forrest, S. R.; Lamansky, S.; Thompson, M. E.; Kwong, R. C. Appl. Phys. Lett. 2001, 78,1622 .

(3) (a) Kober, E. M.; Caspar, J. V.; Lumpkin, R. S.; Meyer, T. J. J. Phys. Chem. 1986, 90, 3722. (b) Perkins, T. A.; Pourreau, D. B.; Netzel, T. L.; Schanze, K. S. J. Phys. Chem. 1989, 93, 4511.

(4) (a) Yeh, S.-J.; Wu, W.-C.; Chen, C.-T.; Song, Y.-H.; Chi, Y.; Ho, M.-H.; Hsu, S.-F.; Chen, C.-H. Adv. Mater. 2005, 17, 285. (b) Ren, X.; Li, J.; Holmes, R. J.; Djurovich, P. I.; Forrest, S. R.; Thompson, M. E. Chem. Mater. 2004, 16, 4743. (c) Karatsu, T.; Nakamura, T.; Yagai, S.; Kitamura, A.; Yamaguchi, K.; Matsushima, Y.; Iwata, T.; Hori, Y.; Hagiwara, T. Chem. Lett. 2003, 32, 886. (d) Holmes, R. J.; D’Andrade, B. W.; Forrest, S. R.; Ren, X.; Li, J.; Thompson, M. E. Appl. Phys. Lett. 2003, 83, 3818. (e) Holmes, R. J.; Forrest, S. R.; Tung, Y.-J.; Kwong, R. C.; Brown, J. J.; Garon, S.; Thompson, M. E. Appl. Phys. Lett. 2003, 82, 2422. (f) Tokito, S.; Iijima, T.; Suzuri, Y.; Kita, H.; Tsuzuki, T.; Sato, F. Appl. Phys. Lett. 2003, 83, 569. (g) Tanaka, I.; Tabata, Y.; Tokito, S. Chem. Phys. Lett. 2004, 400, 86.

(5) (a) Anderson, P. A.; Keene, F. R.; Meyer, T. J.; Moss, J. A.; Strouse, G. F.; Treadway, J. A. J. Chem. Soc., Dalton Trans. 2002, 3820. (b) Koike, K.; Okoshi, N.; Hori, H.; Takeuchi, K.; Ishitani, O.; Tsubaki, H.; Clark, I. P.; George, M. W.; Johnson, F. P. A.; Turner, J. J. J. Am. Chem. Soc. 2002, 124, 11448. (c) Zalis, S.; Farrell, I. R.; Vlcek, A. J. Am. Chem. Soc. 2003, 125, 4580 .

(6) (a) Lee, C.-L.; Das, R. R.; Kim, J.-J. Chem. Mater. 2004, 16, 4642. (b) Nazeeruddin, M. K.; Humphry-Baker, R.; Berner, D.; Rivier, S.; Zuppiroli, L.; Graetzel, M. J. Am. Chem. Soc. 2003, 125, 8790.
Chart 1

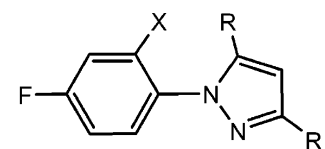

H(C^N) X $=F, R=H ;(d f p z) H$

$X=H, R=H ;(f p z) H$

$X=F, R=M e ;(d f m p z) H$

$X=\mathrm{H}, \mathrm{R}=\mathrm{Me} ;(\mathrm{fmpz}) \mathrm{H}$

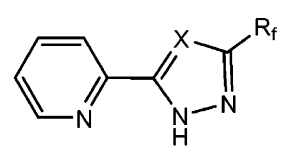

$\left(\mathbf{N}^{\wedge} \mathrm{N}\right) \mathrm{H} \quad X=\mathrm{CH}, \mathrm{R}_{\mathrm{f}}=\mathrm{CF}_{3} ;(\mathrm{fppz}) \mathrm{H}$

$X=N, R_{f}=C_{3} ;(f p t z) H$

$X=N, R_{f}=C_{3} F_{7} ;(h p t z) H$ of tuning the properties of the lowest excited state chemically by employing different ancillary ligands. ${ }^{7}$

Herein, we demonstrate the synthesis and characterization of heteroleptic cyclometalated iridium complexes modified by two distinctive chelating ligands (Chart 1). The synthetic transformation involving these ligands is shown by the following equations:

$$
\begin{gathered}
2 \mathrm{IrCl}_{3}+4 \mathrm{H}\left(\mathrm{C}^{\wedge} \mathrm{N}\right) \rightarrow\left[\left(\mathrm{C}^{\wedge} \mathrm{N}\right)_{2} \operatorname{IrCl}\right]_{2}+4 “ \mathrm{HCl} ” \\
{\left[\left(\mathrm{C}^{\wedge} \mathrm{N}\right)_{2} \operatorname{IrCl}\right]_{2}+2\left(\mathrm{~N}^{\wedge} \mathrm{N}\right) \mathrm{Na} \rightarrow 2\left[\left(\mathrm{C}^{\wedge} \mathrm{N}\right)_{2} \operatorname{Ir}\left(\mathrm{N}^{\wedge} \mathrm{N}\right)\right]+2 \mathrm{NaCl}}
\end{gathered}
$$

The first series of ligands, denoted as $\mathrm{H}\left(\mathrm{C}^{\wedge} \mathrm{N}\right)$, consist of $\mathrm{N}$-phenyl-substituted pyrazoles, which are expected to show good reactivity with $\mathrm{IrCl}_{3} \cdot n \mathrm{H}_{2} \mathrm{O}$ using their phenyl segment to afford products $\left[\left(\mathrm{C}^{\wedge} \mathrm{N}\right)_{2} \operatorname{IrCl}\right]_{2}$ or $\left[\operatorname{Ir}\left(\mathrm{C}^{\wedge} \mathrm{N}\right)_{3}\right]\left(\mathrm{C}^{\wedge} \mathrm{N}=\right.$ cyclometalated ligands). The reaction pattern should be fully consistent with the results documented in earlier papers. ${ }^{8,9}$ Interestingly, the photophysical study of the meridional isomer of $\left[\operatorname{Ir}(\mathrm{dfpz})_{3}\right]((\mathrm{dfpz}) \mathrm{H}=$ (2,4-difluorophenyl)pyrazole) showed an emission peak wavelength of $412 \mathrm{~nm}$ at 77 $\mathrm{K},{ }^{\mathrm{gb}}$ suggesting that Ir complexes incorporating these Fsubstituted $N$-phenylpyrazoles may be suitable for the generation of blue phosphorescence at room temperature due to their exceedingly high energy gaps.

Moreover, as elaborated in the literature,$^{10}$ the second 2-pyridylpyrazole (or triazole), denoted as $\left(\mathrm{N}^{\wedge} \mathrm{N}\right) \mathrm{H}$, possesses a highly acidic $\mathrm{NH}$ fragment. Thus, they may display reaction patterns similar to those of typical anionic chelating ligands such as $\beta$-diketonate or picolate, ${ }^{11}$ giving heteroleptic complexes with a formula of $\left[\left(\mathrm{C}^{\wedge} \mathrm{N}\right)_{2} \operatorname{Ir}\left(\mathrm{N}^{\wedge} \mathrm{N}\right)\right]$ upon addition to the chloride bridged dimer $\left[\left(\mathrm{C}^{\wedge} \mathrm{N}\right)_{2} \operatorname{IrCl}\right]_{2}$. In addition to

(7) Li, J.; Djurovich, P. I.; Alleyne, B. D.; Yousufuddin, M.; Ho, N. N.; Thomas, J. C.; Peters, J. C.; Bau, R.; Thompson, M. E. Inorg. Chem. 2005, 44, 1713.

(8) Dedeian, K.; Shi, J.; Shepherd, N.; Forsythe, E.; Morton, D. C. Inorg. Chem. 2005, 44, 4445.

(9) (a) Lamansky, S.; Djurovich, P.; Murphy, D.; Abdel-Razzaq, F.; Lee, H.-E.; Adachi, C.; Burrows, P. E.; Forrest, S. R.; Thompson, M. E. J. Am. Chem. Soc. 2001, 123, 4304. (b) Tamayo, A. B.; Alleyne, B. D.; Djurovich, P. I.; Lamansky, S.; Tsyba, I.; Ho, N. N.; Bau, R.; Thompson, M. E. J. Am. Chem. Soc. 2003, 125, 7377.

(10) (a) Passaniti, P.; Browne, W. R.; Lynch, F. C.; Hughes, D.; Nieuwenhuyzen, M.; James, P.; Maestri, M.; Vos, J. G. J. Chem. Soc. Dalton Trans. 2002, 1740. (b) Wang, R.; Vos, J. G.; Schmehl, R. H.; Hage, R. J. Am. Chem. Soc. 1992, 114, 1964.

(11) (a) DeRosa, M. C.; Hodgson, D. J.; Enright, G. D.; Dawson, B.; Evans, C. E. B.; Crutchley, R. J. J. Am. Chem. Soc. 2004, 126, 7619. (b) Brooks, J.; Babayan, Y.; Lamansky, S.; Djurovich, P. I.; Tsyba, I.; Bau, R.; Thompson, M. E. Inorg. Chem. 2002, 41, 3055. (c) Lamansky, S.; Djurovich, P.; Murphy, D.; Abdel-Razzaq, F.; Kwong, R.; Tsyba, I.; Bortz, M.; Mui, B.; Bau, R.; Thompson, M. E. Inorg. Chem. 2001, 40, 1704. 
their excellent chelating properties, the asymmetric character of these ligands is extended to the HOMO and LUMO of the metal complexes and, hence, would afford a subtle color tuning via substituting either pyrazolate or triazolate sites. ${ }^{12}$ On these bases, it is thus anticipated that room-temperature blue phosphorescence may be achieved by attaching both of these specially designed $\mathrm{H}\left(\mathrm{C}^{\wedge} \mathrm{N}\right) \mathrm{N}$-phenylpyrazole and $\left(\mathrm{N}^{\wedge} \mathrm{N}\right) \mathrm{H}$ 2-pyridylpyrazole chromophores to the central iridium metal atom.

\section{Experimental Section}

General Information and Materials. All reactions were performed under a nitrogen atmosphere using anhydrous solvents or solvents treated with an appropriate drying reagent. Mass spectra were obtained on a JEOL SX-102A instrument operating in electron impact (EI) mode or fast atom bombardment (FAB) mode. ${ }^{1} \mathrm{H}$ and ${ }^{19} \mathrm{~F}$ NMR spectra were recorded on Varian Mercury-400 or INOVA500 instruments. Elemental analyses were conducted at the NSC Regional Instrumentation Center at National Chiao Tung University. The nitrogen chelates, 3-(trifluoromethyl)-5-(2-pyridyl)pyrazole (fppz)H, 3-(trifluoromethyl)-5-(2-pyridyl)triazole (fptz)H, and 3-(heptafluoropropyl)-5-(2-pyridyl)triazole (hptz)H, were prepared using methods reported in the literature. ${ }^{13}$

Electrochemical Measurement. Cyclic voltammetry (CV) measurements were performed using a BAS $100 \mathrm{~B} / \mathrm{W}$ electrochemical analyzer. The oxidation and reduction measurements were recorded using $\mathrm{Pt}$ wire and an $\mathrm{Au}$ disk coated with $\mathrm{Hg}$ as working electrodes, respectively, in anhydrous $\mathrm{CH}_{2} \mathrm{Cl}_{2}$ and anhydrous THF containing $0.1 \mathrm{M}\left(\mathrm{TBA} \mathrm{PF}_{6}\right.$ as the supporting electrolyte, at a scan rate of 50 $\mathrm{mV} \mathrm{s}^{-1}$. The potentials were measured against an $\mathrm{Ag} / \mathrm{Ag}^{+}(0.01$ $\mathrm{M} \mathrm{AgNO}_{3}$ ) reference electrode with ferrocene as the internal standard.

Synthesis of (fmpz)H. In a $50 \mathrm{~mL}$ reaction flask, 4-(fluorophenyl)hydrazine hydrochloride $(1.56 \mathrm{~g}, 9.6 \mathrm{~mol})$ and acetylacetone $(0.97$ $\mathrm{g}, 9.71 \mathrm{~mol})$ were refluxed in a 1:1 mixture of ethanol and 1,2dichloroethane $(40 \mathrm{~mL})$. After $10 \mathrm{~h}$, the mixture was cooled to room temperature and the solvent removed under vacuum. A $30 \mathrm{~mL}$ volume of ethyl acetate was added to dissolve the product mixture, and the resulting solution was washed with water $(3 \times 30 \mathrm{~mL})$, dried over anhydrous $\mathrm{Na}_{2} \mathrm{SO}_{4}$, and concentrated to dryness under reduced pressure. Finally, this mixture was further purified by silica gel column chromatography (acetate and hexane $=1: 1$ ), giving $1.65 \mathrm{~g}$ of 1-(4-fluorophenyl)-3,5-dimethylpyrazole (fmpzH) as a light brown viscous liquid, yield $89 \%$. The analogous (dfmpz)H was prepared using a similar procedure.

Selected spectral data for (fmpz)H: ${ }^{1} \mathrm{H} \mathrm{NMR}\left(400 \mathrm{MHz}, \mathrm{CDCl}_{3}\right.$, $294 \mathrm{~K}) \delta 7.69\left(\mathrm{~d}, J_{\mathrm{HH}}=8.4 \mathrm{~Hz}, 2 \mathrm{H}\right), 7.57\left(\mathrm{~d}, J_{\mathrm{HH}}=8.8 \mathrm{~Hz}, 2 \mathrm{H}\right)$, $6.02(\mathrm{~s}, 1 \mathrm{H}), 2.34(\mathrm{~s}, 3 \mathrm{H}), 2.28(\mathrm{~s}, 3 \mathrm{H})$. Selected spectral data for (dfmpz)H: ${ }^{1} \mathrm{H}$ NMR (400 MHz, $\left.\mathrm{CDCl}_{3}, 294 \mathrm{~K}\right) \delta 7.37-7.43(\mathrm{~m}$, H), 6.92-6.98 (m, 2H), 5.96 (s, 1H), 2.27 (s, 3H), 2.14 (s, 3H).

Preparation of $\left[(\mathbf{f m p z})_{2} \mathbf{I r C l}\right]_{2}$. The chloride-bridged complex $\left[(\mathrm{fmpz})_{2} \mathrm{IrCl}\right]_{2}$ was synthesized from treatment of $\mathrm{IrCl}_{3} \cdot 3 \mathrm{H}_{2} \mathrm{O}(0.81$ $\mathrm{g}, 2.32 \mathrm{mmol}$ ) with 1-(4-fluorophenyl)-3,5-dimethylpyrazole (fmpzH, $0.98 \mathrm{~g}, 5.13 \mathrm{mmol})$ in 2-ethoxyethanol $(40 \mathrm{~mL})$ for $24 \mathrm{~h}$. The solution was treated with water $(40 \mathrm{~mL})$ to induce precipitation of a colorless solid. The solid product was then filtered out, washed with diethyl ether, and dried under vacuum (1.12 g, $0.92 \mathrm{mmol}$,

(12) Cheng, C.-C.; Yu, W.-S.; Chou, P.-T.; Peng, S.-M.; Lee, G.-H.; Wu, P.-C.; Song, Y.-H.; Chi, Y. Chem. Commun. 2003, 2628.

(13) (a) Kubota, S.; Uda, M.; Nakagawa, T. J. Heterocycl. Chem. 1975, 12, 855. (b) Funabiki, K.; Noma, N.; Kuzuya, G.; Matsui, M.; Shibata, K. J. Chem. Res., Miniprint 1999, 1301.
$79 \%)$. The complexes $\left[(\mathrm{dfmpz})_{2} \mathrm{IrCl}\right]_{2}$ and $\left[(\mathrm{fpz})_{2} \mathrm{IrCl}\right]_{2}$ were prepared using similar conditions.

Preparation of $\left[(\mathbf{d f p z})_{2} \mathbf{I r}(\mathbf{f p p z})\right]$ (1a). A mixture of $\left[(\mathrm{dfpz})_{2} \mathrm{IrCl}\right]_{2}$ (62 mg, $0.053 \mathrm{mmol}$ ), 3-(trifluoromethyl)-5-(2-pyridyl)pyrazole (fppzH, $28 \mathrm{mg}, 0.13 \mathrm{mmol}$ ), and $\mathrm{Na}_{2} \mathrm{CO}_{3}(56 \mathrm{mg}, 0.53 \mathrm{mmol})$ in 2-ethoxyethanol $(20 \mathrm{~mL})$ was heated to reflux for $12 \mathrm{~h}$. An excess of water was added after the solution was cooled to RT (room temperature). The precipitate was collected by filtration and washed with anhydrous ethanol $(10 \mathrm{~mL})$, followed by diethyl ether $(10 \mathrm{~mL})$. Colorless crystals of $\left[(\mathrm{dfpz})_{2} \operatorname{Ir}(\mathrm{fppz})\right]$ (1a) were obtained from cooling the mixed solution of $\mathrm{CH}_{2} \mathrm{Cl}_{2}$ and methanol at room temperature $(61 \mathrm{mg}, 0.079 \mathrm{mmol}, 75 \%)$. The related complexes $\left[(\mathrm{fpz})_{2} \operatorname{Ir}(\mathrm{fppz})\right](\mathbf{1 b}),\left[(\mathrm{dfmpz})_{2} \operatorname{Ir}(\mathrm{fppz})\right](\mathbf{1 c})$, and [(fmpz $\left.)_{2} \operatorname{Ir}(\mathrm{fppz})\right]$ (1d) were prepared using similar procedures, yields 56-65\%.

Spectral Data for 1a. MS (FAB) (obsd $\mathrm{m} / \mathrm{z}$ [assignment]): 764 $\left[\mathrm{M}^{+}\right], 552\left[\mathrm{M}^{+}-\mathrm{fppz}\right] .{ }^{1} \mathrm{H} \mathrm{NMR}\left(500 \mathrm{MHz}, \mathrm{CDCl}_{3}, 294 \mathrm{~K}\right): \delta$ $8.23\left(\mathrm{dd}, J_{\mathrm{HF}}=16.5,16.0 \mathrm{~Hz}, 2 \mathrm{H}\right), 7.87\left(\mathrm{~d}, J_{\mathrm{HH}}=5.5 \mathrm{~Hz}, 1 \mathrm{H}\right)$, $7.76 \sim 7.80(\mathrm{~m}, 2 \mathrm{H}), 7.06\left(\mathrm{t}, J_{\mathrm{HH}}=5.8 \mathrm{~Hz}, 1 \mathrm{H}\right), 6.96(\mathrm{~s}, 1 \mathrm{H})$, $6.86\left(\mathrm{~d}, J_{\mathrm{HH}}=1.5 \mathrm{~Hz}, 1 \mathrm{H}\right), 6.76\left(\mathrm{~d}, J_{\mathrm{HH}}=2 \mathrm{~Hz}, 1 \mathrm{H}\right), 6.51-6.44$ $(\mathrm{m}, 4 \mathrm{H}), 5.76\left(\mathrm{dd}, J_{\mathrm{HH}}=8.0,7.5 \mathrm{~Hz}, 1 \mathrm{H}\right), 5.68\left(\mathrm{dd}, J_{\mathrm{HH}}=8.5,8.0\right.$ $\mathrm{Hz}, 1 \mathrm{H}) .{ }^{19} \mathrm{~F}$ NMR $\left(470 \mathrm{MHz}, \mathrm{CDCl}_{3}, 294 \mathrm{~K}\right): \delta-125.6\left(\mathrm{dd}, J_{\mathrm{HF}}\right.$ $\left.=12.4, J_{\mathrm{FF}}=6.2 \mathrm{~Hz}, 1 \mathrm{~F}\right),-124.8\left(\mathrm{dd}, J_{\mathrm{HF}}=11.5, J_{\mathrm{FF}}=5.7 \mathrm{~Hz}\right.$, $1 \mathrm{~F}),-113.8\left(\mathrm{dd}, J_{\mathrm{HF}}=12.1, J_{\mathrm{FF}}=7.5 \mathrm{~Hz}, 1 \mathrm{~F}\right),-113.2\left(\mathrm{dd}, J_{\mathrm{HF}}\right.$ $\left.=12.8, J_{\mathrm{FF}}=6.5 \mathrm{~Hz}, 1 \mathrm{~F}\right),-60.3(\mathrm{~s}, 3 \mathrm{~F})$. Anal. Calcd for $\mathrm{C}_{27} \mathrm{H}_{15} \mathrm{~F}_{7-}$ $\mathrm{IrN}_{7}$ : N, 12.86; C, 42.52; H, 1.98. Found: N, 12.89; C, 42.28; H, 2.05 .

Spectral Data for 1b. MS (FAB) (obsd $\mathrm{m} / \mathrm{z}$ [assignment]): 728 $\left[\mathrm{M}^{+}\right], 516\left[\mathrm{M}^{+}-\mathrm{fppz}\right] .{ }^{1} \mathrm{H} \mathrm{NMR}\left(400 \mathrm{MHz}, \mathrm{CDCl}_{3}, 294 \mathrm{~K}\right): \delta$ $7.99\left(\mathrm{~d}, J_{\mathrm{HH}}=2.8 \mathrm{~Hz}, 1 \mathrm{H}\right), 7.94\left(\mathrm{~d}, J_{\mathrm{HH}}=2.4 \mathrm{~Hz}, 1 \mathrm{H}\right), 7.91(\mathrm{~d}$, $\left.J_{\mathrm{HH}}=5.6 \mathrm{~Hz}, 1 \mathrm{H}\right), 7.77(\mathrm{~s}, 2 \mathrm{H}), 7.20-7.15(\mathrm{~m}, 2 \mathrm{H}), 7.05(\mathrm{~s}, 1 \mathrm{H})$, $6.97(\mathrm{~s}, 1 \mathrm{H}), 6.90(\mathrm{~s}, 1 \mathrm{H}), 6.74(\mathrm{~s}, 1 \mathrm{H}), 6.70\left(\mathrm{td}, J_{\mathrm{HH}}=8.4,6.0\right.$ $\mathrm{Hz}, 1 \mathrm{H}), 6.64\left(\mathrm{td}, J_{\mathrm{HH}}=8.4,6.0 \mathrm{~Hz}, 1 \mathrm{H}\right), 6.44\left(\mathrm{~d}, J_{\mathrm{HH}}=11.6 \mathrm{~Hz}\right.$, $2 \mathrm{H}), 5.97\left(\mathrm{dd}, J_{\mathrm{HH}}=8.8,2.8 \mathrm{~Hz}, 1 \mathrm{H}\right), 5.90\left(\mathrm{dd}, J_{\mathrm{HH}}=7.4,2.8\right.$ $\mathrm{Hz}, 1 \mathrm{H}) .{ }^{19} \mathrm{~F} \mathrm{NMR}\left(470 \mathrm{MHz}, \mathrm{CDCl}_{3}, 294 \mathrm{~K}\right): \delta-116.6(\mathrm{~s}, 1 \mathrm{~F})$, -116.2 (s, 1F), -60.1 (s, 3F). Anal. Calcd for $\mathrm{C}_{27} \mathrm{H}_{17} \mathrm{~F}_{5} \operatorname{IrN}_{7}: \mathrm{N}$, 13.49; C, 44.63; H, 2.36. Found: N, 13.31; C, 44.42; H, 2.21.

Spectral Data for 1c. MS (FAB) (obsd $\mathrm{m} / z$ [assignment]): 820 $\left[\mathrm{M}^{+}\right], 608\left[\mathrm{M}^{+}-\mathrm{fppz}\right] .{ }^{1} \mathrm{H}$ NMR $\left(400 \mathrm{MHz}, \mathrm{CDCl}_{3}, 294 \mathrm{~K}\right): \delta$ $7.72-7.65(\mathrm{~m}, 2 \mathrm{H}), 7.45\left(\mathrm{~d}, J_{\mathrm{HH}}=5.6 \mathrm{~Hz}, 1 \mathrm{H}\right), 6.98-6.94(\mathrm{~m}$, $1 \mathrm{H}), 6.98-6.50(\mathrm{~m}, 1 \mathrm{H}), 6.46-6.40(\mathrm{~m}, 1 \mathrm{H}), 5.99(\mathrm{~s}, 1 \mathrm{H}), 5.94$ $\left(\mathrm{dd}, J_{\mathrm{HH}}=8.2,2.4 \mathrm{~Hz}, 1 \mathrm{H}\right), 5.91(\mathrm{~s}, 1 \mathrm{H}), 5.46\left(\mathrm{dd}, J_{\mathrm{HH}}=7.4,2.8\right.$ $\mathrm{Hz}, 1 \mathrm{H}), 2.65\left(\mathrm{~d}, J_{\mathrm{HH}}=4 \mathrm{~Hz}, 3 \mathrm{H}\right), 2.62\left(\mathrm{~d}, J_{\mathrm{HH}}=4.4 \mathrm{~Hz}, 3 \mathrm{H}\right)$, $1.51(\mathrm{~s}, 3 \mathrm{H}), 1.45$ (s, 3H). ${ }^{19} \mathrm{~F}$ NMR $\left(470 \mathrm{MHz}, \mathrm{CDCl}_{3}, 294 \mathrm{~K}\right)$ : $\delta-115.6(\mathrm{~s}, 1 \mathrm{~F}),-114.6(\mathrm{~s}, 1 \mathrm{~F}),-112.9(\mathrm{~s}, 1 \mathrm{~F}),-112.6(\mathrm{~s}, 1 \mathrm{~F})$, -60.1 (s, 3F). Anal. Calcd for $\mathrm{C}_{31} \mathrm{H}_{23} \mathrm{~F}_{7} \mathrm{IrN}_{7}$ : N, 11.97; C, 45.47; H, 2.83. Found: N, 12.10; C, 45.38; H, 2.67.

Spectral Data for 1d. MS (FAB) (obsd $\mathrm{m} / \mathrm{z}$ [assignment]): 785 $\left[\mathrm{M}^{+}\right], 571\left[\mathrm{M}^{+}-\right.$fppz]. ${ }^{1} \mathrm{H}$ NMR $\left(500 \mathrm{MHz}, \mathrm{CDCl}_{3}, 294 \mathrm{~K}\right): \delta$ $7.73(\mathrm{~s}, 1 \mathrm{H}), 7.66\left(\mathrm{~d}, J_{\mathrm{HH}}=5.5 \mathrm{~Hz}, 2 \mathrm{H}\right), 7.34-7.31(\mathrm{~m}, 2 \mathrm{H}), 6.97$ (s, 2H), $6.67 \sim 6.59(\mathrm{~m}, 2 \mathrm{H}), 6.02\left(\mathrm{dd}, J_{\mathrm{HH}}=7.5,2.5 \mathrm{~Hz}, 1 \mathrm{H}\right)$, $5.92\left(\mathrm{~d}, J_{\mathrm{HF}}=14.5 \mathrm{~Hz}, 2 \mathrm{H}\right), 5.85\left(\mathrm{dd}, J_{\mathrm{HH}}=10.0,3.0 \mathrm{~Hz}, 1 \mathrm{H}\right)$, 2.75 (s, 3H), 2.72 (s, 3H), 1.53 (s, 3H), 1.46 (s, 3H). ${ }^{19} \mathrm{~F}$ NMR (470 MHz, $\mathrm{CD}_{2} \mathrm{Cl}_{2}, 294 \mathrm{~K}$ ): $\delta-118.4(\mathrm{~s}, 1 \mathrm{~F}),-117.9(\mathrm{~s}, 1 \mathrm{~F})$, -59.9 (s, 3F). Anal. Calcd for $\mathrm{C}_{31} \mathrm{H}_{25} \mathrm{~F}_{5} \mathrm{IrN}_{7}$ : N, 12.53; C, 47.56; H, 3.22. Found: N, 12.42; C, 47.31; H, 3.30.

Preparation of $\left[(\mathbf{d f p z})_{2} \mathbf{I r}(\mathbf{f p t z})\right]$ (2a). Procedures identical with those for 1 were followed, using $60 \mathrm{mg}$ of $\left[(\mathrm{dfpz})_{2} \mathrm{IrCl}\right]_{2}(0.052$ $\mathrm{mmol}), 27 \mathrm{mg}$ of triazole fptzH $(0.13 \mathrm{mmol})$, and $56 \mathrm{mg}$ of $\mathrm{Na}_{2}-$ $\mathrm{CO}_{3}(0.53 \mathrm{mmol})$ in $20 \mathrm{~mL}$ of 2-ethoxyethanol. Colorless crystals of $\left[(\mathrm{dfpz})_{2} \operatorname{Ir}(\mathrm{fptz})\right]$ (2a) were obtained from crystallization using a mixture of $\mathrm{CH}_{2} \mathrm{Cl}_{2}$ and methanol at room temperature (66 mg, 0.086 mmol, $83 \%$ ). 
Spectral Data for 2a. MS (FAB) (obsd $\mathrm{m} / \mathrm{z}$ [assignment]): 765 $\left[\mathrm{M}^{+}\right], 552\left[\mathrm{M}^{+}-\right.$fptz]. ${ }^{1} \mathrm{H}$ NMR $\left(500 \mathrm{MHz}, \mathrm{CDCl}_{3}, 294 \mathrm{~K}\right): \delta$ $8.28-8.33(\mathrm{~m}, 3 \mathrm{H}), 7.91-7.93(\mathrm{~m}, 2 \mathrm{H}), 7.24\left(\mathrm{td}, J_{\mathrm{HH}}=6.5,1.0\right.$ $\mathrm{Hz}, 1 \mathrm{H}), 6.96\left(\mathrm{~d}, J_{\mathrm{HH}}=0.8 \mathrm{~Hz}, 1 \mathrm{H}\right), 6.73\left(\mathrm{~d}, J_{\mathrm{HH}}=2.0 \mathrm{~Hz}, 1 \mathrm{H}\right)$, $6.61\left(\mathrm{ddd}, J_{\mathrm{HF}}=11.5,9.0,2.5 \mathrm{~Hz}, 1 \mathrm{H}\right), \delta 6.52 \sim 6.55(\mathrm{~m}, 1 \mathrm{H})$, $6.61\left(\mathrm{dd}, J_{\mathrm{HH}}=3.0,2.5 \mathrm{~Hz}, J_{\mathrm{HH}}=3.0,1.6 \mathrm{~Hz}, 2 \mathrm{H}\right), 5.78\left(\mathrm{dd}, J_{\mathrm{HH}}\right.$ $=8.0,3.0 \mathrm{~Hz}, 1 \mathrm{H}), 5.67\left(\mathrm{dd}, J_{\mathrm{HH}}=8.3,2.3 \mathrm{~Hz}, 1 \mathrm{H}\right) .{ }^{19} \mathrm{~F}$ NMR $\left(470 \mathrm{MHz}, \mathrm{CDCl}_{3}, 294 \mathrm{~K}\right): \delta-125.3\left(\mathrm{dd}, J_{\mathrm{HF}}=11.7, J_{\mathrm{FF}}=5.6\right.$ $\mathrm{Hz}, 1 \mathrm{~F}),-124.6\left(\mathrm{dd}, J_{\mathrm{HF}}=11.9, J_{\mathrm{FF}}=6.6 \mathrm{~Hz}, 1 \mathrm{~F}\right),-113.5(\mathrm{dd}$, $\left.J_{\mathrm{HF}}=15.1, J_{\mathrm{FF}}=8.5 \mathrm{~Hz}, 1 \mathrm{~F}\right),-112.8\left(\mathrm{dd}, J_{\mathrm{HF}}=13.8, J_{\mathrm{FF}}=6.6\right.$ $\mathrm{Hz}, 1 \mathrm{~F}),-63.7$ (s, 3F). Anal. Calcd for $\mathrm{C}_{26} \mathrm{H}_{14} \mathrm{~F}_{7} \mathrm{IrN}_{8}$ : N, 14.67; C, 40.89; H, 1.85. Found: N, 14.61; C, 40.94; H, 1.90.

Preparation of $\left.\left[(\mathbf{f m p z})_{2} \mathbf{I r}(\mathbf{f p t z})\right] \mathbf{( 2 d}\right)$. Procedures identical with those for 1 were followed, using $120 \mathrm{mg}$ of $\left[(\mathrm{fmpz})_{2} \mathrm{IrCl}\right]_{2}(0.099$ mmol), $53 \mathrm{mg}$ of fptzH $(0.248 \mathrm{mmol})$, and $105 \mathrm{mg}$ of $\mathrm{Na}_{2} \mathrm{CO}_{3}(0.99$ mmol) in $25 \mathrm{~mL}$ of 2-methoxyethanol. Colorless crystals of [(fmpz $\left.)_{2} \operatorname{Ir}(\mathrm{fptz})\right](\mathbf{2 d})$ were obtained from cooling the mixed solution of $\mathrm{CH}_{2} \mathrm{Cl}_{2}$ and methanol at room temperature $(50 \mathrm{mg}, 0.065 \mathrm{mmol}$, $33 \%$ ).

Spectral Data for 2d. MS (FAB) (obsd $m / z$ [assignment]): 784 $\left[\mathrm{M}^{+}\right], 571\left[\mathrm{M}^{+}-\right.$fptz]. ${ }^{1} \mathrm{H}$ NMR $\left(500 \mathrm{MHz}, \mathrm{CDCl}_{3}, 294 \mathrm{~K}\right): \delta$ $8.50(\mathrm{~s}, 1 \mathrm{H}), 7.86\left(\mathrm{t}, J_{\mathrm{HH}}=8.5 \mathrm{~Hz}, 1 \mathrm{H}\right), 7.74\left(\mathrm{~d}, J_{\mathrm{HH}}=5.0 \mathrm{~Hz}\right.$, $1 \mathrm{H}), 7.37-7.30(\mathrm{~m}, 2 \mathrm{H}), 7.17\left(\mathrm{t}, J_{\mathrm{HH}}=6.0 \mathrm{~Hz}, 1 \mathrm{H}\right), 6.69\left(\mathrm{td}, J_{\mathrm{HH}}\right.$ $=9.5,8.5 \mathrm{~Hz}, 2 \mathrm{H}), 6.04\left(\mathrm{dd}, J_{\mathrm{HH}}=10.0,3.5 \mathrm{~Hz}, 1 \mathrm{H}\right), 5.95\left(\mathrm{~d}, J_{\mathrm{HF}}\right.$ $=14.0 \mathrm{~Hz}, 2 \mathrm{H}), 5.86\left(\mathrm{dd}, J_{\mathrm{HH}}=10.0,2.5 \mathrm{~Hz}, 1 \mathrm{H}\right), 2.75(\mathrm{~s}, 3 \mathrm{H})$, 2.74 (s, 3H), 1.54 (s, 3H), 1.44 (s, 3H). ${ }^{19} \mathrm{~F} \mathrm{NMR}\left(470 \mathrm{MHz}, \mathrm{CD}_{2}-\right.$ $\mathrm{Cl}_{2}, 294 \mathrm{~K}$ ): $\delta-118.2(\mathrm{~s}, 1 \mathrm{~F}),-117.5$ (s, 1F), -63.2 (s, 3F). Anal. Calcd for $\mathrm{C}_{29} \mathrm{H}_{23} \mathrm{~F}_{5} \mathrm{IrN}_{8}$ : N, 14.30; C, 45.97; H, 3.09. Found: $\mathrm{N}$, 14.13; C, 45.45; H, 3.37.

Preparation of $\left[(\mathbf{d f p z})_{2} \mathbf{I r}(\mathbf{h p t z})\right]$ (3a). Procedures identical with those for 1 were followed, using $225 \mathrm{mg}$ of $\left[(\mathrm{dfpz})_{2} \mathrm{IrCl}\right]_{2}(0.19$ $\mathrm{mmol}), 133 \mathrm{mg}$ of triazole hptzH $(0.42 \mathrm{mmol})$, and $202 \mathrm{mg}$ of $\mathrm{Na}_{2}-$ $\mathrm{CO}_{3}(1.9 \mathrm{mmol})$ in $40 \mathrm{~mL}$ of 2-ethoxyethanol. Colorless crystals of $\left[(\mathrm{dfpz})_{2} \mathrm{Ir}(\mathrm{hptz})\right]$ (3a) were obtained from crystallization using a mixture of $\mathrm{CH}_{2} \mathrm{Cl}_{2}$ and methanol at room temperature $(257 \mathrm{mg}$, $0.297 \mathrm{mmol}, 78 \%)$. The complexes [(fpz $\left.)_{2} \operatorname{Ir}(\mathrm{hptz})\right](\mathbf{3 b}),\left[(\mathrm{dfmpz})_{2} \mathrm{Ir}-\right.$ (hptz)] (3c), and [(fmpz) $\left.)_{2} \operatorname{Ir}(\mathrm{hptz})\right]$ (3d) were prepared using similar procedures, yields $72-81 \%$.

Spectral Data for 3a. MS (FAB) (obsd $m / z$ [assignment]): 865 $\left[\mathrm{M}^{+}\right], 552\left[\mathrm{M}^{+}-\mathrm{hptz}\right] .{ }^{1} \mathrm{H} \mathrm{NMR}\left(500 \mathrm{MHz}, \mathrm{CDCl}_{3}, 294 \mathrm{~K}\right): \delta$ $8.26-8.33(\mathrm{~m}, 3 \mathrm{H}), 7.89-7.92(\mathrm{~m}, 2 \mathrm{H}), 7.24\left(\mathrm{td}, J_{\mathrm{HH}}=6.5,1.0\right.$ $\mathrm{Hz}, 1 \mathrm{H}), 6.85\left(\mathrm{~d}, J_{\mathrm{HH}}=2.5 \mathrm{~Hz}, 1 \mathrm{H}\right), 6.74\left(\mathrm{~d}, J_{\mathrm{HH}}=2.0 \mathrm{~Hz}, 1 \mathrm{H}\right)$, $6.61\left(\mathrm{ddd}, J_{\mathrm{HH}}=11.5,9.0,2.5 \mathrm{~Hz}, 1 \mathrm{H}\right), 6.48 \sim 6.53(\mathrm{~m}, 1 \mathrm{H})$, $6.45\left(\mathrm{t}, J_{\mathrm{HH}}=2.5 \mathrm{~Hz}, 2 \mathrm{H}\right), 5.79\left(\mathrm{dd}, J_{\mathrm{HH}}=8.0,7.5 \mathrm{~Hz}, 1 \mathrm{H}\right), 5.64$ $\left(\mathrm{dd}, J_{\mathrm{HH}}=8.5,8.0 \mathrm{~Hz}, 1 \mathrm{H}\right) .{ }^{19} \mathrm{~F} \mathrm{NMR}\left(470 \mathrm{MHz}, \mathrm{CDCl}_{3}, 294 \mathrm{~K}\right)$ : $\delta-126.6(\mathrm{~s}, 2 \mathrm{~F}),-125.2\left(\mathrm{dd}, J_{\mathrm{HF}}=13.2, J_{\mathrm{FF}}=10.8 \mathrm{~Hz}, 1 \mathrm{~F}\right)$, $-124.2\left(\mathrm{dd}, J_{\mathrm{HF}}=12.6, J_{\mathrm{FF}}=10.8 \mathrm{~Hz}, 1 \mathrm{~F}\right),-113.3\left(\mathrm{dd}, J_{\mathrm{HF}}=\right.$ $\left.15.1, J_{\mathrm{FF}}=12.6 \mathrm{~Hz}, 1 \mathrm{~F}\right),-112.4\left(\mathrm{dd}, J_{\mathrm{HF}}=15.1, J_{\mathrm{FF}}=13.1 \mathrm{~Hz}\right.$, $1 \mathrm{~F}),-111.8\left(\mathrm{~d}, J_{\mathrm{FF}}=13.1 \mathrm{~Hz}, 2 \mathrm{~F}\right),-80.2\left(\mathrm{t}, J_{\mathrm{FF}}=8.4 \mathrm{~Hz}, 3 \mathrm{~F}\right)$. Anal. Calcd for $\mathrm{C}_{28} \mathrm{H}_{14} \mathrm{~F}_{11} \mathrm{IrN}_{8}$ : N, 12.97; C, 38.94; H, 1.63. Found: N, 12.89; C, 38.89; H, 1.72 .

Spectral Data for 3b. MS (FAB) (obsd $m / z$ [assignment]): 829 $\left[\mathrm{M}^{+}\right], 515\left[\mathrm{M}^{+}-\mathrm{hptz}\right] .{ }^{1} \mathrm{H}$ NMR $\left(500 \mathrm{MHz}\right.$, acetone- $\left.d_{6}, 294 \mathrm{~K}\right)$ : $\delta 8.60\left(\mathrm{dd}, J_{\mathrm{HH}}=11.0,10.7 \mathrm{~Hz}, 2 \mathrm{H}\right), 8.24\left(\mathrm{~d}, J_{\mathrm{HH}}=8.0,1 \mathrm{H}\right)$, $8.01 \sim 8.15(\mathrm{~m}, 2 \mathrm{H}), 7.65\left(\mathrm{dd}, J_{\mathrm{HH}}=9.0,8.5 \mathrm{~Hz}, 1 \mathrm{H}\right), 7.54(\mathrm{dd}$, $\left.J_{\mathrm{HH}}=9.0,8.5 \mathrm{~Hz}, 1 \mathrm{H}\right), 7.47\left(\mathrm{td}, J_{\mathrm{HH}}=7.5,5.7 \mathrm{~Hz}, 1 \mathrm{H}\right), 7.17(\mathrm{~d}$, $\left.J_{\mathrm{HH}}=2.5 \mathrm{~Hz}, 1 \mathrm{H}\right), 6.87\left(\mathrm{~d}, J_{\mathrm{HH}}=2.0 \mathrm{~Hz}, 1 \mathrm{H}\right), 6.78\left(\mathrm{td}, J_{\mathrm{HH}}=\right.$ $10.0,7.0 \mathrm{~Hz}, 1 \mathrm{H}), 6.36-6.87(\mathrm{~m}, 3 \mathrm{H}), 6.02\left(\mathrm{dd}, J_{\mathrm{HH}}=9.0,8.7\right.$ $\mathrm{Hz}, 1 \mathrm{H}), 5.89$ (dd, $\left.J_{\mathrm{HH}}=9.5,8.5 \mathrm{~Hz}, 1 \mathrm{H}\right) .{ }^{19} \mathrm{~F} \mathrm{NMR}(470 \mathrm{MHz}$, $\left.\mathrm{CDCl}_{3}, 294 \mathrm{~K}\right): \delta-126.7(\mathrm{~s}, 2 \mathrm{~F}),-117.8\left(\mathrm{~d}, J_{\mathrm{FF}}=4.2 \mathrm{~Hz}, 1 \mathrm{~F}\right)$, $-116.7\left(\mathrm{~d}, J_{\mathrm{FF}}=4.2 \mathrm{~Hz}, 1 \mathrm{~F}\right),-110.8(\mathrm{~s}, 2 \mathrm{~F}),-80.5\left(\mathrm{t}, J_{\mathrm{FF}}=8.4\right.$ $\mathrm{Hz}, 3 \mathrm{~F})$. Anal. Calcd for $\mathrm{C}_{28} \mathrm{H}_{16} \mathrm{~F}_{9} \mathrm{IrN}_{8}$ : N, 13.54; C, 40.63; H,
Table 1. Crystal Data and Refinement Parameters for Complexes 1a and 3d

\begin{tabular}{|c|c|c|}
\hline param & $1 \mathrm{a}$ & $3 d$ \\
\hline formula & $\mathrm{C}_{27} \mathrm{H}_{15} \mathrm{~F}_{7} \mathrm{~N}_{7} \mathrm{Ir}$ & $\mathrm{C}_{34} \mathrm{H}_{28} \mathrm{~F}_{9} \mathrm{~N}_{8} \mathrm{Cl}_{4} \mathrm{Ir}$ \\
\hline$M_{\mathrm{r}}$ & 762.66 & 1053.64 \\
\hline cryst system & monoclinic & triclinic \\
\hline space group & $P 2_{1} / c$ & $P \overline{1}$ \\
\hline cryst size $\left(\mathrm{mm}^{3}\right)$ & $0.3 \times 0.3 \times 0.15$ & $0.25 \times 0.2 \times 0.2$ \\
\hline$a(\AA)$ & $10.3424(4)$ & $9.3973(2)$ \\
\hline$b(\AA)$ & $12.9104(5)$ & $12.6736(5)$ \\
\hline$c(\AA)$ & 19.8601(7) & $17.8433(7)$ \\
\hline$\alpha(\operatorname{deg})$ & & $103.941(1)$ \\
\hline$\beta(\mathrm{deg})$ & 104.316(9) & $99.917(1)$ \\
\hline$\gamma(\mathrm{deg})$ & & 107.633(1) \\
\hline$V\left(\AA^{3}\right)$ & $2569.46(2)$ & $1895.27(1)$ \\
\hline$Z$ & 4 & 2 \\
\hline$\rho_{\text {calc }}\left(\mathrm{g} / \mathrm{cm}^{3}\right)$ & 1.972 & 1.846 \\
\hline temp $(\mathrm{K})$ & $295(2)$ & $150(1)$ \\
\hline$\mu\left(\mathrm{mm}^{-1}\right)$ & 5.280 & 3.888 \\
\hline reflens colled & 25271 & 25080 \\
\hline indepndt reflcns & $5904[R(\mathrm{int})=0.0360]$ & $8685[R$ (int $)=0.0299]$ \\
\hline$R_{F}, R_{\mathrm{w}}\left(F^{2}\right)$ (all data) ${ }^{a}$ & $0.0348,0.0696$ & $0.0302,0.0687$ \\
\hline$R_{F}, R_{\mathrm{W}}\left(F^{2}\right)[I>2 \sigma(I)]^{a}$ & $0.0274,0.0663$ & $0.0279,0.0673$ \\
\hline GOF & 1.017 & 1.490 \\
\hline
\end{tabular}

1.95. Found: N, 13.27; C, 40.79; H, 2.01. Anal. Calcd for $\mathrm{C}_{28} \mathrm{H}_{16} \mathrm{~F}_{9}$ $\mathrm{IrN}_{8}$ : N, 13.54; C, 40.63; H, 1.95. Found: N, 13.41; C, 40.58; H, 2.01.

Spectral Data for 3c. MS (FAB) (obsd $\mathrm{m} / \mathrm{z}$ [assignment]): 921 $\left[\mathrm{M}^{+}\right], 608\left[\mathrm{M}^{+}-\mathrm{hptz}\right] .{ }^{1} \mathrm{H}$ NMR $\left(400 \mathrm{MHz}, \mathrm{CDCl}_{3}, 294 \mathrm{~K}\right): \delta$ $8.34\left(\mathrm{~d}, J_{\mathrm{HH}}=8.0 \mathrm{~Hz}, 1 \mathrm{H}\right), 7.84\left(\mathrm{td}, J_{\mathrm{HH}}=7.8,1.2 \mathrm{~Hz}, 1 \mathrm{H}\right), 7.56-$ $7.54(\mathrm{~m}, 1 \mathrm{H}), 7.18\left(\mathrm{ddd}, J_{\mathrm{HH}}=7.2,2.0,1.6 \mathrm{~Hz}, 1 \mathrm{H}\right), 6.59-6.53$ $(\mathrm{m}, 1 \mathrm{H}), 6.48-6.41(\mathrm{~m}, 1 \mathrm{H}), 6.02-6.00(\mathrm{~m}, 2 \mathrm{H}), 5.91(\mathrm{~s}, 1 \mathrm{H})$, $5.41\left(\mathrm{dd}, J_{\mathrm{HH}}=6.0,2.4 \mathrm{~Hz}, 1 \mathrm{H}\right), 2.66\left(\mathrm{~d}, J_{\mathrm{HH}}=12.0 \mathrm{~Hz}, 3 \mathrm{H}\right)$, $2.62\left(\mathrm{~d}, J_{\mathrm{HH}}=12.0 \mathrm{~Hz}, 3 \mathrm{H}\right), 1.51(\mathrm{~s}, 3 \mathrm{H}), 1.40(\mathrm{~s}, 3 \mathrm{H})$. Anal. Calcd for $\mathrm{C}_{32} \mathrm{H}_{22} \mathrm{~F}_{11} \mathrm{IrN}_{8}$ : N, 12.18; C, 41.79; $\mathrm{H}, 2.41$. Found: $\mathrm{N}, 12.24$; C, 41.82; H, 2.12.

Spectral Data for 3d. MS (FAB) (obsd $\mathrm{m} / z$ [assignment]): 885 $\left[\mathrm{M}^{+}\right], 571\left[\mathrm{M}^{+}-\mathrm{hptz}\right] .{ }^{1} \mathrm{H} \mathrm{NMR}\left(400 \mathrm{MHz}, \mathrm{CD}_{2} \mathrm{Cl}_{2}, 294 \mathrm{~K}\right): \delta$ $8.26\left(\mathrm{~d}, J_{\mathrm{HH}}=7.88 \mathrm{~Hz}, 1 \mathrm{H}\right), 7.92\left(\mathrm{td}, J_{\mathrm{HH}}=7.8,1.7 \mathrm{~Hz}, 1 \mathrm{H}\right)$, $7.81\left(\mathrm{~d}, J_{\mathrm{HH}}=5.3 \mathrm{~Hz}, 1 \mathrm{H}\right), 7.44 \sim 7.48(\mathrm{~m}, 2 \mathrm{H}), 7.20\left(\mathrm{t}, J_{\mathrm{HH}}=\right.$ $5.5 \mathrm{~Hz}, 1 \mathrm{H}), 6.70-6.79(\mathrm{~m}, 2 \mathrm{H}), 6.14\left(\mathrm{dd}, J_{\mathrm{HH}}=8.9,6.5 \mathrm{~Hz}, 1 \mathrm{H}\right)$, $6.04\left(\mathrm{~d}, J_{\mathrm{HH}}=3.8 \mathrm{~Hz}, 2 \mathrm{H}\right), 5.98\left(\mathrm{dd}, J_{\mathrm{HH}}=9.3,9.2 \mathrm{~Hz}, 1 \mathrm{H}\right), 2.80$ $\left(\mathrm{d}, J_{\mathrm{HH}}=8.4 \mathrm{~Hz}, 6 \mathrm{H}\right), 1.53\left(\mathrm{~d}, J_{\mathrm{HH}}=8.2 \mathrm{~Hz}, 6 \mathrm{H}\right) .{ }^{19} \mathrm{~F} \mathrm{NMR}(470$ $\left.\mathrm{MHz}, \mathrm{CDCl}_{3}, 294 \mathrm{~K}\right): \delta-127.3(\mathrm{~s}, 2 \mathrm{~F}),-118.7(\mathrm{~m}, 1 \mathrm{~F}),-118.4$ $(\mathrm{m}, 1 \mathrm{~F}),-111.6(\mathrm{~m}, 2 \mathrm{~F}),-81.1\left(\mathrm{t}, J_{\mathrm{FF}}=8.6 \mathrm{~Hz}, 3 \mathrm{~F}\right)$. Anal. Calcd for $\mathrm{C}_{32} \mathrm{H}_{24} \mathrm{~F}_{9} \mathrm{IrN}_{8}$ : N, 12.68; C, 43.49; H, 2.74. Found: N, 12.57; C, 43.62; H, 2.69.

X-ray Structural Determination. Single-crystal X-ray diffraction data were recorded on a Bruker SMART CCD diffractometer using $\lambda(\mathrm{Mo} \mathrm{K} \alpha)$ radiation $(\lambda=0.71073 \AA)$. The data collection was executed using the SMART program. Cell refinement and data reduction were performed with the SAINT program. The structure was determined using the SHELXTL/PC program and refined using full-matrix least squares. All non-hydrogen atoms were refined anisotropically, whereas hydrogen atoms were placed at the calculated positions and included in the final stage of refinements with fixed positional parameters. The crystallographic refinement parameters of complexes $\mathbf{1 a}$ and $\mathbf{3 d}$ are summarized in Table 1, while the selective bond distances and angles of these complexes are listed in Tables 2 and 3, respectively.

Spectral Measurement. Steady-state absorption and emission spectra were recorded with a Hitachi (U-3310) spectrophotometer and an Edinburgh (FS920) fluorometer, respectively. Quinine sulfate with an emission yield of $\Phi \sim 0.57\left(\lambda_{\max } \sim 460 \mathrm{~nm}\right)$ in $0.1 \mathrm{M}$ 
Table 2. Selected Bond Length $(\AA)$ and Angles (deg) for Complex 1a

\begin{tabular}{|c|c|c|c|}
\hline $\mathrm{Ir}-\mathrm{N}(1)$ & 2.004(3) & $\mathrm{Ir}-\mathrm{N}(6)$ & $2.096(3)$ \\
\hline $\mathrm{Ir}-\mathrm{N}(3)$ & $2.013(3)$ & $\mathrm{Ir}-\mathrm{C}(1)$ & $2.018(3)$ \\
\hline $\mathrm{Ir}-\mathrm{N}(5)$ & $2.149(3)$ & $\mathrm{Ir}-\mathrm{C}(10)$ & $2.011(4)$ \\
\hline $\mathrm{N}(1)-\mathrm{Ir}-\mathrm{N}(3)$ & $173.17(13)$ & $\mathrm{N}(5)-\mathrm{Ir}-\mathrm{C}(10)$ & $169.22(14)$ \\
\hline $\mathrm{N}(6)-\mathrm{Ir}-\mathrm{C}(1)$ & $172.31(13)$ & $\mathrm{N}(1)-\mathrm{Ir}-\mathrm{C}(1)$ & $80.06(14)$ \\
\hline $\mathrm{N}(3)-\mathrm{Ir}-\mathrm{C}(10)$ & $80.17(16)$ & $\mathrm{N}(5)-\mathrm{Ir}-\mathrm{N}(6)$ & $76.82(11)$ \\
\hline $\mathrm{Ir}-\mathrm{N}(1)$ & $2.033(3)$ & $\mathrm{Ir}-\mathrm{N}(6)$ & $2.123(3)$ \\
\hline $\mathrm{Ir}-\mathrm{N}(3)$ & $2.024(3)$ & $\mathrm{Ir}-\mathrm{C}(11)$ & $2.006(3)$ \\
\hline $\mathrm{Ir}-\mathrm{N}(5)$ & $2.150(3)$ & $\mathrm{Ir}-\mathrm{C}(22)$ & $2.010(3)$ \\
\hline $\mathrm{N}(1)-\mathrm{Ir}-\mathrm{N}(3)$ & $173.09(10)$ & $\mathrm{N}(6)-\mathrm{Ir}-\mathrm{C}(22)$ & 170.71(11) \\
\hline $\mathrm{N}(5)-\mathrm{Ir}-\mathrm{C}(11)$ & $179.78(12)$ & $\mathrm{N}(1)-\mathrm{Ir}-\mathrm{C}(11)$ & $79.82(12)$ \\
\hline $\mathrm{N}(3)-\mathrm{Ir}-\mathrm{C}(22)$ & 79.91(13) & $\mathrm{N}(5)-\mathrm{Ir}-\mathrm{N}(6)$ & $76.25(10)$ \\
\hline
\end{tabular}

$\mathrm{H}_{2} \mathrm{SO}_{4}$ served as a standard to calculate the emission quantum yield. An integrating sphere (Labsphere) was applied to measure the quantum yield in the solid state, in which the solid sample film was prepared via the vapor deposition method and excited by a $365 \mathrm{~nm} \mathrm{Ar}{ }^{+}$laser line. The resulting luminescence was acquired with a charge-coupled detector (Princeton Instruments, model CCD1100) for subsequent quantum yield analyses. Nanosecond lifetime studies were performed with an Edinburgh FL 900 photon-counting system with a hydrogen-filled/or a nitrogen lamp as the excitation source. Data were analyzed using the nonlinear least-squares procedure in combination with an iterative convolution method. The emission decays were analyzed by the sum of exponential functions, which allows partial removal of the instrument time broadening and consequently renders a temporal resolution of $\sim 200$ ps.

Computational Methodology. Calculations on the electronic ground state of complexes $\mathbf{1 - 3}$ were carried out using B3LYP density functional theory. ${ }^{14,15}$ A "double- $\zeta$ " quality basis set consisting of Hay and Wadt's effective core potentials (LANL2DZ) ${ }^{16}$ was employed for Ir atom, and a $6-31 \mathrm{G}^{*}$ basis, ${ }^{17}$ for $\mathrm{H}, \mathrm{C}, \mathrm{N}$, and $\mathrm{F}$ atoms. A relativistic effective core potential (ECP) replaced the inner core electrons of $\operatorname{Ir}(\mathrm{III})$, leaving the outer core $\left(5 \mathrm{~s}^{2} 5 \mathrm{p}^{6}\right)$ electrons and the $5 \mathrm{~d}^{6}$ valence electrons. Time-dependent DFT (TDDFT) calculations ${ }^{18}$ using the B3LYP functional were then performed on the basis of the structural optimized geometries. Typically, the lowest 10 triplet and 10 singlet roots of the nonhermitian eigenvalue equations were obtained to determine the vertical excitation energies. Oscillator strengths were deduced from the dipole transition matrix elements (for singlet states only). The ground-state B3LYP and excited-state TDDFT calculations were carried out using Gaussian03. ${ }^{19}$

\section{Results and Discussion}

Synthesis and Characterization. Condensation of fluorinesubstituted phenylhydrazine hydrochloride with acetylacetone or with 1,1,3,3-tetramethoxypropane gave formation of four $\mathrm{H}\left(\mathrm{C}^{\wedge} \mathrm{N}\right)$ pyrazole ligands, cf. 1-(2,4-difluorophenyl)pyrazole (dfpz)H, 1-(4-fluorophenyl)pyrazole (fpz)H, 1-(2,4-difluo-

(14) Lee, C.; Yang, W.; Parr, R. G. Phys. Rev. B 1988, 37, 785.

(15) Becke, A. D. J. Chem. Phys. 1993, 98, 5648.

(16) (a) Hay, P. J.; Wadt, W. R. J. Chem. Phys. 1985, 82, 270. (b) Wadt, W. R.; Hay, P. J. J. Chem. Phys. 1985, 82, 284. (c) Hay, P. J.; Wadt, W. R. J. Chem. Phys. 1985, 82, 299.

(17) Hariharan, P. C.; Pople, J. A. Mol. Phys. 1974, 27, 209.

(18) (a) Jamorski, C.; Casida, M. E.; Salahub, D. R. J. Chem. Phys. 1996, 104, 5134. (b) Petersilka, M.; Grossmann, U. J.; Gross, E. K. U. Phys. Rev. Lett. 1996, 76, 1212. (c) Bauernschmitt, R.; Ahlrichs, R.; Hennrich, F. H.; Kappes, M. M. J. Am. Chem. Soc. 1998, 120, 5052. (d) Casida, M. E. J. Chem. Phys. 1998, 108, 4439. (e) Stratmann, R. E.; Scuseria, G. E.; Frisch, M. J. J. Chem. Phys. 1998, 109, 8218.

\section{Chart 2}
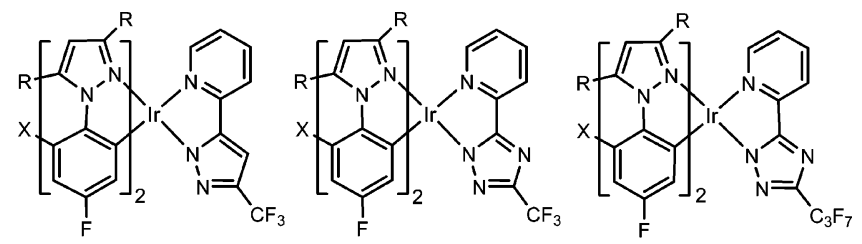

$$
\begin{aligned}
& \text { (1a) } X=F, R=H \\
& \text { (1b) } X=H, R=H \\
& \text { (1c) } X=F, R=M e \\
& \text { (1d) } X=H, R=M e
\end{aligned}
$$

(2a) $X=F, R=H$

(3a) $X=F, R=H$

(3b) $X=H, R=H$

(3b) $X=F, R=M e$

(3d) $X=H, R=M e$

rophenyl)-3,5-dimethylpyrazole (dfmpz)H, and 1-(4-fluorophenyl)-3,5-dimethylpyrazole (fmpz)H, in good yields. ${ }^{20}$ Subsequently, treatment of these $\mathrm{C}^{\wedge} \mathrm{N}$ cyclometalating ligands with $\mathrm{IrCl}_{3} \cdot n \mathrm{H}_{2} \mathrm{O}$ in refluxing ethoxyethanol afforded the respective chloride-bridged iridium complexes $\left[(\mathrm{dfpz})_{2}-\right.$ $\mathrm{IrCl}]_{2},\left[(\mathrm{fpz})_{2} \mathrm{IrCl}\right]_{2},\left[(\mathrm{dfmpz})_{2} \mathrm{IrCl}\right]_{2}$, and $\left[(\mathrm{fmpz})_{2} \mathrm{IrCl}\right]_{2}$. The anticipated heteroleptic complexes, [(dfpz $\left.)_{2} \operatorname{Ir}(\mathrm{fppz})\right](\mathbf{1 a})$, $\left[(\mathrm{fpz})_{2} \operatorname{Ir}(\mathrm{fppz})\right](\mathbf{1 b}),\left[(\mathrm{dfmpz})_{2} \operatorname{Ir}(\mathrm{fppz})\right](\mathbf{1 c})$, and $\left[(\mathrm{fmpz})_{2} \operatorname{Ir}-\right.$ (fppz)] (1d), in which (fppz)H = 3-(trifluoromethyl)-5-(2pyridyl)pyrazole, were then prepared from reactions involving these chloride-bridged complexes and the designated $\left(\mathrm{N}^{\wedge} \mathrm{N}\right) \mathrm{H}$ ligand such as $\mathrm{CF}_{3}$-substituted pyridylpyrazole $(\mathrm{fppz}) \mathrm{H}$ in the presence of $\mathrm{Na}_{2} \mathrm{CO}_{3}$ as a proton scavenger. A similar synthetic strategy was applied to synthesize other analogues incorporating the triazole ligands, such as 3-(trifluoromethyl)-5-(2-pyridyl)triazole (fptz)H and 3-(heptafluoropropyl)-5-(2-pyridyl)triazole (hptz)H. These reactions then afforded two more series of iridium complexes: [ $\left.\mathrm{L}_{2} \operatorname{Ir}(\mathrm{fptz})\right]$ (2) and $\left[\mathrm{L}_{2}{ }_{2} \operatorname{Ir}(\mathrm{hptz})\right]$ (3) (L and $\mathrm{L}^{\prime}=\mathrm{dfpz}, \mathrm{fpz}$, dfmpz, and fmpz). Chart 2 summarizes their structural drawings and numberings. It is notable that these iridium complexes are moderately soluble in chlorinated solvents, particularly for the ones with the methyl-substituted $N$-phenylpyrazole ligands or with the heptafluoropropyl substituent. They also show great thermal stability, as supported by negligible degradation in solution phase over a long period of time. Detailed characterizations were carried out using routine MS and ${ }^{1} \mathrm{H}$ and ${ }^{19} \mathrm{~F}$ NMR spectroscopy and finally with elemental analysis, among which complexes $\mathbf{1 a}$ and $\mathbf{3 a}$ were further identified using single-crystal X-ray analysis to establish their three-dimensional structures.

(19) Frisch, M. J.; Trucks, G. W.; Schlegel, H. B.; Scuseria, G. E.; Robb, M. A.; Cheeseman, J. R.; Montgomery, J. A., Jr.; Vreven, T.; Kudin, K. N.; Burant, J. C.; Millam, J. M.; Iyengar, S. S.; Tomasi, J.; Barone, V.; Mennucci, B.; Cossi, M.; Scalmani, G.; Rega, N.; Petersson, G. A.; Nakatsuji, H.; Hada, M.; Ehara, M.; Toyota, K.; Fukuda, R.; Hasegawa, J.; Ishida, M.; Nakajima, T.; Honda, Y.; Kitao, O.; Nakai, H.; Klene, M.; Li, X.; Knox, J. E.; Hratchian, H. P.; Cross, J. B.; Bakken, V.; Adamo, C.; Jaramillo, J.; Gomperts, R.; Stratmann, R. E.; Yazyev, O.; Austin, A. J.; Cammi, R.; Pomelli, C.; Ochterski, J. W.; Ayala, P. Y.; Morokuma, K.; Voth, G. A.; Salvador, P.; Dannenberg, J. J.; Zakrzewski, V. G.; Dapprich, S.; Daniels, A. D.; Strain, M. C.; Farkas, O.; Malick, D. K.; Rabuck, A. D.; Raghavachari, K.; Foresman, J. B.; Ortiz, J. V.; Cui, Q.; Baboul, A. G.; Clifford, S.; Cioslowski, J.; Stefanov, B. B.; Liu, G.; Liashenko, A.; Piskorz, P.; Komaromi, I.; Martin, R. L.; Fox, D. J.; Keith, T.; Al-Laham, M. A.; Peng, C. Y.; Nanayakkara, A.; Challacombe, M.; Gill, P. M. W.; Johnson, B.; Chen, W.; Wong, M. W.; Gonzalez, C.; Pople, J. A. Gaussian 03, revision C.02; Gaussian, Inc.: Wallingford, CT, 2004.

(20) (a) Nonoyama, M. J. Organomet. Chem. 1975, 86, 263. (b) Mukherjee, R. Coord. Chem. Rev. 2000, 203, 151. 


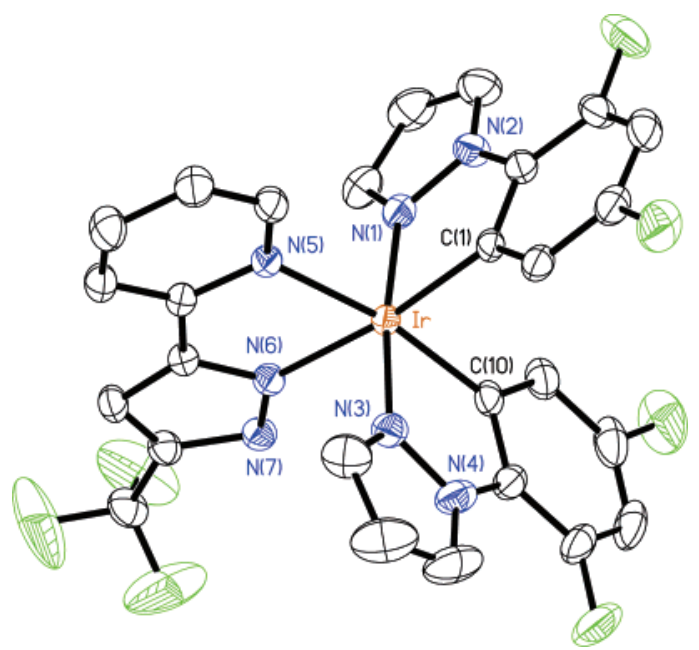

Figure 1. ORTEP diagram of complex 1a with thermal ellipsoids shown at $30 \%$ probability level.

As depicted in Figure 1, complex 1a reveals a distorted octahedral geometry around the Ir atom with two cyclometalated dfpz $\left(\mathrm{C}^{\wedge} \mathrm{N}\right)$ ligands and one anionic fppz $\left(\mathrm{N}^{\wedge} \mathrm{N}\right)$ ligand. All three chelate ligands show small bite angles of $76.8-80.2^{\circ}$ and caused the observed structural distortion. The dfpz ligands adopt mutually an eclipsed configuration with the nitrogen atoms $\mathrm{N}(1)$ and $\mathrm{N}(3)$ residing at the trans locations, and the $\mathrm{Ir}-\mathrm{N}$ distances lie between 2.004(3) and 2.013(3) A. The cyclometalated carbon atoms C(1) and C(10) are mutually cis on the iridium and show similar distances, 2.018(3) and 2.011(4) $\AA$. The third fppz ligand displays slightly elongated $\mathrm{Ir}-\mathrm{N}$ distances of 2.096(3) and 2.149(3) $\AA$ vs those of the trans-orientated $\mathrm{Ir}-\mathrm{N}$ distances of the fppz ligands. This is believed to be caused by the stronger $\mathrm{Ir}-\mathrm{C}$ bonding interaction of the dfpz ligands, which eventually weakened the $\mathrm{Ir}-\mathrm{N}$ bonds at their trans-disposition. Moreover, the overall structural arrangement is similar to several previously reported examples, including chloride-bridged dimer complex $\left[(\mathrm{ppy})_{2} \mathrm{IrCl}\right]_{2},{ }^{21}$ the diketonate complexes such as $\left[(\mathrm{ppy})_{2} \operatorname{Ir}(\mathrm{acac})\right]$ and others, ${ }^{22}$ and even the derivatives with pyrazolyl ancillary ligands, ${ }^{23}$ confirming that the third fppz ligand entered into the iridium coordination sphere via a simple ligand substitution.

Moreover, the dimethyl-substituted fmpz complex 3d showed an essentially identical structural arrangement (Figure 2). The most notable difference is the slightly elongated $\mathrm{Ir}-\mathrm{N}$ distances of the cyclometalated fmpz ligands, $\mathrm{Ir}-\mathrm{N}(1)=$ 2.033(3) and $\operatorname{Ir}-\mathrm{N}(3)=2.024(3) \AA$, the results of which could be ascribed to an increase in steric interaction between the methyl group of fmpz ligands and the unique hptz ligand. However, this nonbonding interaction seems to be small, so no apparent influence on the $\mathrm{N}(1)-\mathrm{Ir}-\mathrm{N}(3)$ bond angle was observed between these two complexes.

Electrochemistry. The electrochemical behavior of these Ir metal complexes was investigated by cyclic voltammetry

(21) Graces, F. O.; King, K. A.; Watts, R. J. Inorg. Chem. 1988, $27,3464$.

(22) (a) Tsuzuki, T.; Shirasawa, N.; Suzuki, T.; Tokito, S. Adv. Mater. 2003, 15, 1455. (b) Laskar, I. R.; Hsu, S.-F.; Chen, T.-M. Polyhedron 2005, 24, 189 .

(23) Li, J.; Djurovich, P. I.; Alleyne, B. D.; Tsyba, I.; Ho, N. N.; Bau, R.; Thompson, M. E. Polyhedron 2004, 23, 419.

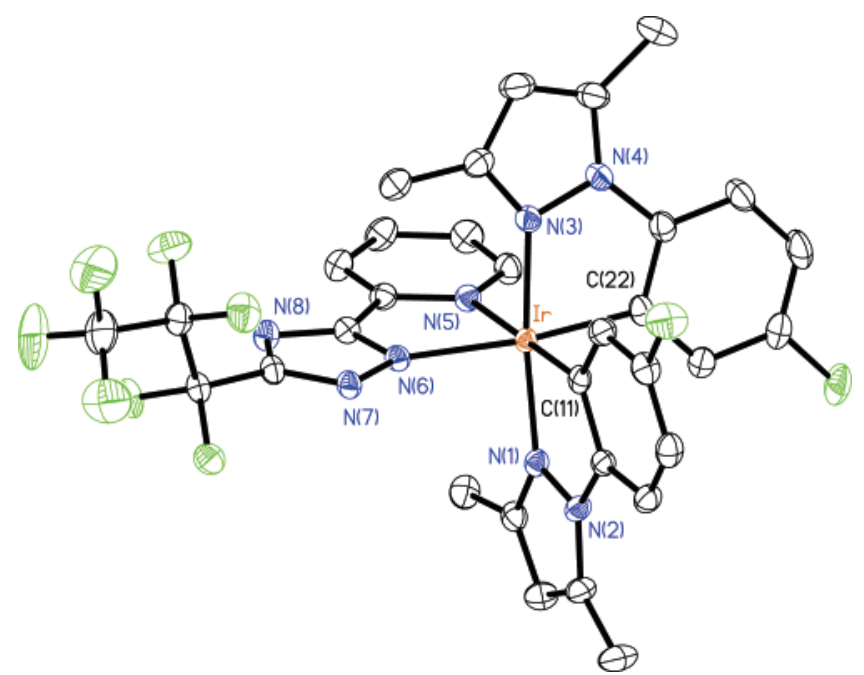

Figure 2. ORTEP diagram of complex 3d with thermal ellipsoids shown at $40 \%$ probability level.

using ferrocene as the internal standard. The results are listed in Table 4. During the anodic scan in $\mathrm{CH}_{2} \mathrm{Cl}_{2}$, all iridium metal complexes exhibited a reversible oxidation with potentials in the region of $0.69-1.11 \mathrm{~V}$. Upon the switch to the cathodic sweep in THF, one irreversible wave, with potentials ranging from -2.58 to $-2.93 \mathrm{~V}$, was detected. It is believed that the oxidation occurred mainly at the Ir metal site, together with minor contributions from the cyclometalated $\mathrm{C}^{\wedge} \mathrm{N}$ ligand and the third $\mathrm{N}^{\wedge} \mathrm{N}$ chelating anion. Accordingly, replacing the 4,6-difluorophenyl with a 4-fluorophenyl moiety and changing from pyrazole to the more electron-donating 3,5-dimethylpyrazole led to the decrease of their oxidation potentials. This is demonstrated by the higher oxidation potential of $1 \mathbf{a}(0.98 \mathrm{~V})$ vs that of complex $\mathbf{1 b}(0.83 \mathrm{~V})$, as well as the decrease of oxidation potentials for complexes $\mathbf{1 c}(0.87 \mathrm{~V})$ and $\mathbf{1 d}(0.69 \mathrm{~V})$ vs those of $\mathbf{1 a}, \mathbf{b}$. Moreover, the oxidation potentials along the series 1a $(0.98$ $\mathrm{V}) \rightarrow \mathbf{2 a}(1.11 \mathrm{~V}) \rightarrow \mathbf{3 a}(1.11 \mathrm{~V})$ and complexes $\mathbf{1 d}(0.69$ $\mathrm{V}) \rightarrow \mathbf{2 d}(0.78 \mathrm{~V}) \rightarrow \mathbf{3 d}(0.78 \mathrm{~V})$ revealed that the pyridyltriazolate ligands (i.e. fptz and hptz) are more electronwithdrawing than is their pyrazolate counterpart fppz.

Furthermore, in contrast to the oxidation process, the reduction may occur primarily on the low-lying $\pi^{*}$ orbitals of the pyridyl segment of the ancillary $\mathrm{N}^{\wedge} \mathrm{N}$ ligand. This assignment is supported by the subsequent DFT calculations and by the fact that the reduction potentials of the phenylpyrazolyl complexes $f a c-\operatorname{Ir}(\mathrm{ppz})_{3}$ and $f a c-\operatorname{Ir}(\mathrm{dfppz})_{3}$ are relatively much greater than those of the pyridyl complexes $f a c-\operatorname{Ir}(\mathrm{ppy})_{3}$ and $f a c-\operatorname{Ir}(46 \mathrm{dfppy})_{3} .{ }^{9 \mathrm{~b}}$ Moreover, the irreversible cathodic peak potentials for the pyrazolate complexes $\mathbf{1 a}-\mathbf{d}$ occur in the range $(-2.72$ to $-2.93 \mathrm{~V})$, which are notably greater than those of the triazolate complexes $\mathbf{2 a}, \mathbf{d}$ and $\mathbf{3 a} \mathbf{a}-\mathbf{d}$ $(-2.53$ to $-2.74 \mathrm{~V})$. This reductive electrochemistry is thus consistent with the pyridylpyrazolate group being significantly more difficult to reduce than its triazolate counterparts.

Photophysical Properties. Figures 3-5 show the UV/ vis absorption and emission spectra of complexes $\mathbf{1}-\mathbf{3}$ in $\mathrm{CH}_{2} \mathrm{Cl}_{2}$ and the corresponding emission in solid crystal at $298 \mathrm{~K}$. To gain more insight into the emission properties, 
Yang et al.

Table 4. Electrochemical Data (V) and Photophysical Properties of All Iridium Complexes ${ }^{a}$

\begin{tabular}{|c|c|c|c|c|c|c|c|c|}
\hline complexes & $E_{1 / 2}{ }^{\mathrm{ox}}\left(\Delta E_{\mathrm{p}}\right)$ & $E_{\mathrm{pc}}^{\text {red }}\left(\Delta E_{\mathrm{p}}\right)$ & $\lambda_{\mathrm{abs}}(\mathrm{nm})$ & $\lambda_{\mathrm{em}}(\mathrm{nm})$ & $10^{-3} \Phi$ & $\tau(\mathrm{ns})$ & $k_{\mathrm{r}}\left(10^{5} \mathrm{~s}^{-1}\right)$ & $\tau_{\text {solid }}(\mathrm{ns})$ \\
\hline $1 a$ & $0.98(100)$ & -2.72 (irr) & $\sim 295,349$ & $455^{b}(451)$ & $6.7^{b}(130)$ & 42.4 & 1.6 & 1834 \\
\hline 1b & $0.83(90)$ & -2.79 (irr) & $\sim 296,348$ & $458(451)$ & 3.5 & 16.2 & 2.2 & 21.8 \\
\hline $1 c$ & $0.87(100)$ & -2.93 (irr) & $\sim 297,352$ & $456(450)$ & 0.11 & 1.2 & 0.9 & 20 \\
\hline 1d & $0.69(80)$ & -2.87 (irr) & $\sim 300,355$ & 457 (457) & 2.1 & 5.1 & 4.1 & 381 \\
\hline $2 a$ & $1.11(100)$ & -2.72 (irr) & $\sim 300,349$ & 457 (448) & 4.6 & 8.6 & 5.4 & 133 \\
\hline 2d & $0.78(110)$ & -2.64 (irr) & $\sim 299,375$ & $460(450)$ & 3.3 & 6.0 & 5.5 & 115 \\
\hline $3 \mathbf{a}$ & $1.11(110)$ & -2.53 (irr) & $\sim 302,348$ & $455(447)$ & 4.4 & 9.3 & 4.7 & 133 \\
\hline $3 \mathbf{b}$ & $0.93(100)$ & -2.58 (irr) & $\sim 306,349$ & $460(459)$ & 8.6 & 8.6 & 10.0 & 63 \\
\hline $3 c$ & $0.96(100)$ & -2.58 (irr) & $\sim 295,345$ & 459 (446) & 0.34 & 0.5 & 6.8 & 20.4 \\
\hline 3d & $0.78(110)$ & -2.74 (irr) & $\sim 302,353$ & 469 (449) & 7.5 & 5.1 & 14.7 & 295 \\
\hline
\end{tabular}

$\operatorname{aEpc}{ }^{\text {red }}\left(\Delta E_{\mathrm{p}}\right)$ represents the irreversible cathodic peak potential. ${ }^{b}$ All photophysical data were recorded in degassed $\mathrm{CH}_{2} \mathrm{Cl}_{2}$ at room temperature, while data in parentheses show the emission data recorded in solid state at room temperature.

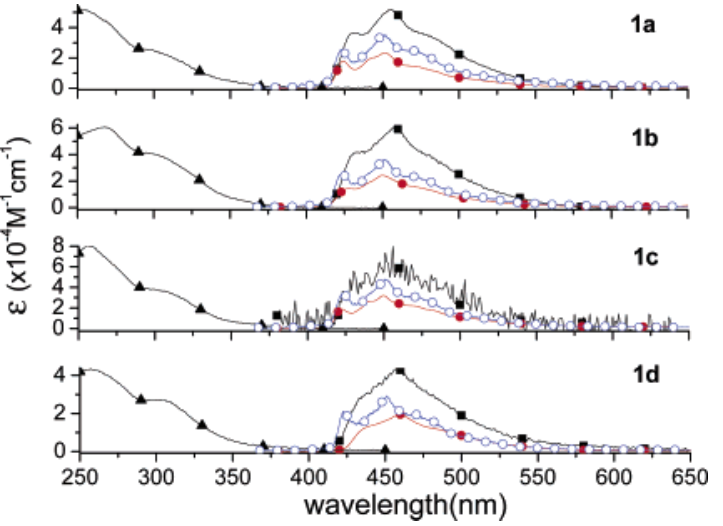

Figure 3. UV/vis absorption ( $\mathbf{\Lambda})$ and emission ( $\mathbf{\square})$ spectra of complexes $\mathbf{1 a}-\mathbf{d}$ in $\mathrm{CH}_{2} \mathrm{Cl}_{2}(298 \mathrm{~K})$. The red trace $(\bullet)$ denotes the emission spectra obtained from solid crystal at room temperature. The blue circle $(O)$ shows the corresponding emission in a $77 \mathrm{~K} \mathrm{CH}_{2} \mathrm{Cl}_{2}$ matrix.

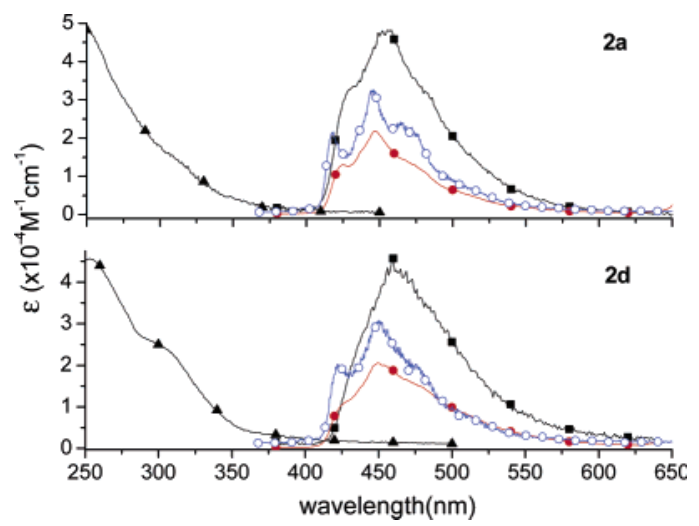

Figure 4. UV/vis absorption (ム) and emission (ם) spectra of complexes 2a,d in $\mathrm{CH}_{2} \mathrm{Cl}_{2}$. The red trace $(\bullet)$ denotes the emission spectra obtained from solid crystal at room temperature. The blue circle $(O)$ shows the corresponding emission in a $77 \mathrm{~K} \mathrm{CH}_{2} \mathrm{Cl}_{2}$ matrix.

the corresponding emission spectra in a $77 \mathrm{~K} \mathrm{CH}_{2} \mathrm{Cl}_{2}$ matrix were also included. In general, the dominant absorption band in the spectral region of $\leq 280 \mathrm{~nm}$, for which $\epsilon$ values at the absorption maximum are calculated to be $\geq 5 \times 10^{3} \mathrm{M}^{-1}$ $\mathrm{cm}^{-1}$, is attributed to the local ${ }^{1} \pi \pi^{*}$ transition of the pyrazolate (or triazolate) fragment and, to a small extent, the phenyl ring (vide infra). As supported in the later theoretical approaches, the origin of broad, structureless bands at $\sim 280-360 \mathrm{~nm}$ for $\mathbf{1}-\mathbf{3}$ can be assigned to a combination of triazolate (or pyrazolate)-to-pyridine intraligand charge transfer (ILCT) and a fluorine atom substituted phenyl-to-pyridine interligand $\pi \pi^{*}$ transition (LLCT) over-

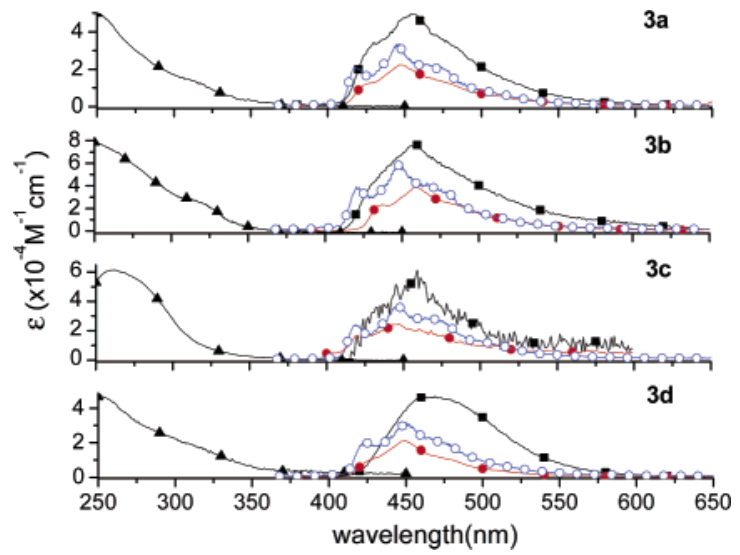

Figure 5. UV/vis absorption (A) and emission (ם) spectra of complexes $3 \mathbf{a}-\mathbf{d}$ in $\mathrm{CH}_{2} \mathrm{Cl}_{2}$. The red trace $(\bullet)$ denotes the emission spectra obtained from solid crystal at room temperature. The blue circle $(O)$ shows the corresponding emission in a $77 \mathrm{~K} \mathrm{CH}_{2} \mathrm{Cl}_{2}$ matrix.

lapping with the $\operatorname{Ir}\left(\mathrm{d}_{\pi}\right)$ to pyridyl ligand transition (MLCT) in the singlet manifold. Moreover, the lower lying band that appears in the region of $>380 \mathrm{~nm}$, as supported by their extinction coefficients of $\ll 1000 \mathrm{M}^{-1} \mathrm{~cm}^{-1}$, is tentatively assigned to the ${ }^{3} \mathrm{MLCT}$, mixed with significant ${ }^{3} \pi \pi$ transition character. For example, the absorptivity of this band, e.g., $\epsilon$ $=200 \mathrm{M}^{-1} \mathrm{~cm}^{-1}$ at the peak wavelength of $\sim 400 \mathrm{~nm}$ for 3d, suggests that the $\mathrm{S}_{0} \rightarrow{ }^{3} \mathrm{MLCT} /{ }^{3} \pi \pi^{*}$ transition is greatly enhanced and becomes partially allowed due to strong spinorbit coupling. As supported by the emission spectral feature, the phosphorescence seems to be dominated by the ${ }^{3} \pi \pi$ transition (vide infra), further detailed assignments for which are firmly supported by theoretical calculations elaborated in the following section.

Complexes 1-3 all exhibit unique blue emission with peak wavelengths at $\sim 450-470 \mathrm{~nm}$ in $\mathrm{CH}_{2} \mathrm{Cl}_{2}$. The emission spectral profile is reminiscent of the Os(II) 2-pyridylpyrazolate (triazolate) complexes ${ }^{24}$ that, however, are less suitable for OLED application due to the presence of carbonyl ligands and shows additional $\sim 5-10 \mathrm{~nm}$ blue shift compared to that of the "almost blue-emitting" $\operatorname{Ir}(\mathrm{III})$ complexes. ${ }^{25}$ Varying the substituents on the ligands, such as adding methyl groups on pyrazole of the $\mathrm{C}^{\wedge} \mathrm{N}$ ligand site (cf. 1a,d), does not show

(24) (a) Yu, J.-K.; Hu, Y.-H.; Cheng, Y.-M.; Chou, P.-T.; Peng, S.-M.; Lee, G.-H.; Carty, A. J.; Tung, Y.-L.; Lee, S.-W.; Chi, Y.; Liu, C.-S. Chem.-Eur. J. 2004, 10, 6255. (b) Wu, P.-C.; Yu, J.-K.; Song, Y.H.; Chi, Y.; Chou, P.-T.; Peng, S.-M.; Lee, G.-H. Organometallics 2003, 22, 4938.

(25) Coppo, P.; Plummer, E. A.; De Cola, L. Chem. Commun. 2004, 1774.

7776 Inorganic Chemistry, Vol. 44, No. 22, 2005 
much effect on the spectral feature in terms of e.g. peak wavelengths and the associated vibronic structures. The results may indicate that pyrazole on the $\mathrm{C}^{\wedge} \mathrm{N}$ site plays a minor role in the lower lying transitions. Likewise, substitution of pyrazole on the $\mathrm{N}^{\wedge} \mathrm{N}$ ligand site of complex 1a by triazole, forming complexes $\mathbf{2 a}$ or $\mathbf{3 a}$, gives rise to slight spectral changes. For the case of free pyridylpyrazolate ligand, the lowest transition normally takes place from pyrazolate (HOMO) to pyridyl sites (LUMO) ${ }^{26}$ Assuming a similar type of transition for triazolate, the decrease of the HOMO level in triazolate due to the addition of a nitrogen atom should result in an appreciable increase of the energy gap. The rather small spectral alternation upon triazole substitution implies that the lower lying states may originate from a mixing of transitions from various ligand chromophores, in which the intraligand pyrazolate (or triazole) to pyridine transition plays only a part in the contribution. Furthermore, in comparison to complex 3a, possessing two fluorine substituents, complex $\mathbf{3 b}$, bearing only one fluorine atom on the $\mathrm{C}^{\wedge} \mathrm{N}$ ligand, undergoes a small bathochromic shift of $\sim 3-5 \mathrm{~nm}$. Accordingly, the cyclometalated phenyl ring, to a certain extent, also contributes to the lowest $\mathrm{T}_{1}-$ $\mathrm{S}_{0}$ transition, in which the fluorine substituent at the metaposition with respect to the metal center acts as an electronwithdrawing group to further lower the HOMO energy level, resulting in an increase of the energy gap. ${ }^{27}$ However, the $<5 \mathrm{kcal} / \mathrm{mol}$ decrease in the phosphorescence gap from difluorine-substituted 3a to monofluorine-substituted complex $\mathbf{3 b}$ is rather small, supporting the proposed mechanism in that the $\mathrm{S}_{0} \rightarrow \mathrm{T}_{1}$ transition may concurrently incorporate various ligand chromophores. Further evidence of these viewpoints is given by computational results (vide infra).

The emission intensities of $\mathbf{1 - 3}$ are all relatively weak, with quantum yields measured to be in the range of $\Phi=$ $(0.1-8.6) \times 10^{-3}$ in degassed $\mathrm{CH}_{2} \mathrm{Cl}_{2}$. The quantum efficiencies of complexes $\mathbf{1 - 3}$ were measured under the condition of $10^{-5-} 10^{-6} \mathrm{M}$, and there was no concentration dependence on the quantum efficiency and the corresponding lifetime. For all cases, negligible changes of the emission intensity were observed upon aerating the $\mathrm{CH}_{2} \mathrm{Cl}_{2}$ solution. The nearly $\mathrm{O}_{2}$-independent emission intensities can be supported by their corresponding emission lifetimes of as short as $<50 \mathrm{~ns}$ in both degassed and aerated solution (see Table 4). Accordingly, a radiative decay rate constant $k_{\mathrm{r}}$ of $\sim 10^{5} \mathrm{~s}^{-1}$ was deduced for $\mathbf{1}-\mathbf{3}$ (e.g., $1.6 \times 10^{5} \mathrm{~s}^{-1}$ for $\mathbf{1 a}$, $5.4 \times 10^{5} \mathrm{~s}^{-1}$ for $\mathbf{2 a}$; see Table 4). The $>1 \mu$ s radiative lifetime (i.e., $k_{\mathrm{r}}<10^{6} \mathrm{~s}^{-1}$ ), in combination with vibronic spectral features, leads us to assign the emission originating from the triplet manifold, in which the ${ }^{3} \pi \pi^{*}$ configuration may have a greater contribution to the transition than that of MLCT. Further support was given by the photophysical measurements in the solid state. In the solid crystal, a slight

(26) (a) Chen, H.-Y.; Chi, Y.; Liu, C.-S.; Yu, J.-K.; Chen, K.-S.; Chou, P.-T.; Peng, S.-M.; Lee, G.-H.; Carty, A. J.; Yeh, S.-J.; Chen, C.-T. Adv. Funct. Mater. 2005, 15, 567.

(27) (a) Grushin, V. V.; Herron, N.; LeCloux, D. D.; Marshall, W. J.; Petrov, V. A.; Wang, Y. Chem. Commun. 2001, 1494. (b) Wang, Y.; Herron, N.; Grushin, V. V.; LeCloux, D.; Petrov, V. Appl. Phys. Lett. 2001, $79,449$. or negligible spectral blue shift was observed for all complexes (see Figures 3-5 and Table 4). Moreover, as depicted in Figures $3-5$, in comparison to that measured in room-temperature solid, the vibronic spectral feature remains unchanged at $77 \mathrm{~K}$ for complexes $\mathbf{1 - 3}$. The results unambiguously conclude that ${ }^{3} \pi \pi$ is the lowest lying triplet state for the studied complexes. In comparison to that in solution, all complexes have the tendency to increase emission yield and hence the observed lifetime in the solid. For complex 1a, the quantum efficiency $\Phi_{\mathrm{p}}$ was measured to $\sim 0.13$, with a lifetime of $1.8 \mu \mathrm{s}$ in the solid state at $298 \mathrm{~K}$. The quantum efficiency of all others was too low $(<0.02)$ to give a reliable value on the basis of the integrating-sphere method (see Experimental Section).

One intriguing feature lies in that the quantum yields of blue-emitting complexes 1-3 are much smaller than most of the highly emissive, red-emitting Ir complexes reported in the literature, for which the pyridylpyrazolate (triazolate) ligands served only as the ancillary $\mathrm{N}^{\wedge} \mathrm{N}$ spectator and the emission is principally derived from the MLCT and $\pi \pi^{*}$ states of the $\mathrm{C}^{\wedge} \mathrm{N}$ cyclometalated ligands of the molecule. ${ }^{28}$ The nonradiative rate constants $k_{\mathrm{nr}}+k_{\mathrm{nr}}(T)$ (vide infra), calculated according to $k_{\mathrm{nr}}+k_{\mathrm{nr}}(T)=\left(k_{\mathrm{r}} / \Phi_{\mathrm{p}}\right)-k_{\mathrm{r}}$, were deduced to be in the range $\sim 10^{8} \mathrm{~s}^{-1}$. Since the heteroleptic (i.e. tris-bidentated) structure of complexes $\mathbf{1}-\mathbf{3}$ is considered to be rigid, a major radiationless deactivation associated with large amplitude skeletal motion seems to be very unlikely. Note that complexes $\mathbf{1}-\mathbf{3}$ show very good photostability. ${ }^{29}$ Thus, the radiationless pathway resulting from any photochemistry ${ }^{30}$ should be considered as a very minor deactivating channel. In addition, both emission quantum yield and observed lifetime are only slightly dependent on pendant substituents such as $\mathrm{Me}, \mathrm{CF}_{3}$, or $\mathrm{C}_{3} \mathrm{~F}_{7}$. Likewise, the role of torsional motion participating in the radiationless deactivation can be discarded. ${ }^{31}$

We further performed temperature-dependent experiments in an attempt to gain insight into the deactivation mechanism on the complexes studied. A prototype used in this study is complex 3d due to its good solubility in methylcyclohexane; a solvent that has a low freezing point of $145 \mathrm{~K}$ is suited to the temperature-dependent approach. As shown in Figure 6, the phosphorescence of $\mathbf{3 d}$ revealed strong temperature dependence. The quantum yield increased from $1.0 \times 10^{-3}$ at $298 \mathrm{~K}$ to a plateau $\left(\Phi_{\mathrm{p}} \sim 0.46\right)$ at $\sim 140 \mathrm{~K}$ and remained constant at lower temperatures. Temperature-dependent relaxation dynamics also correlate well with respect to the steady-state approaches. The lifetime followed the steady-

(28) (a) Song, Y.-H.; Yeh, S.-J.; Chen, C.-T.; Chi, Y.; Liu, C.-S.; Yu, J.K.; Hu, Y.-H.; Chou, P.-T.; Peng, S.-M.; Lee, G.-H. Adv. Funct. Mater. 2004, 14, 1221. (b) Hwang, F.-M.; Chen, H.-Y.; Chen, P.-S.; Liu, C.-S.; Chi, Y.; Shu, C.-F.; Wu, F.-I.; Chou, P.-T.; Peng, S.-M.; Lee, G.-H. Inorg. Chem. 2005, 44, 1344.

(29) In an experiment using $355 \mathrm{~nm}$ (3rd harmonic of Nd:YAG laser, 10 $\mathrm{mJ} /\left(\mathrm{cm}^{2} /\right.$ pulse) $(10 \mathrm{~Hz}$ repetition rate) as an excitation source to photolyze 1a in $\mathrm{CH}_{2} \mathrm{Cl}_{2}$ for a period of $10 \mathrm{~min}$, negligible photoproducts were produced, as indicated by a single spot (complex 1a) in the TLC plate. Similar results were observed for other complexes.

(30) Lumpkin, R. S.; Kober, E. M.; Worl, L. A.; Murtaza, Z.; Meyer, T. J. J. Phys. Chem. 1990, 94, 239.

(31) For recent example, see: Metzger, B. S.; Spangler, L. H. J. Phys. Chem. A 1997, 101, 5431. 


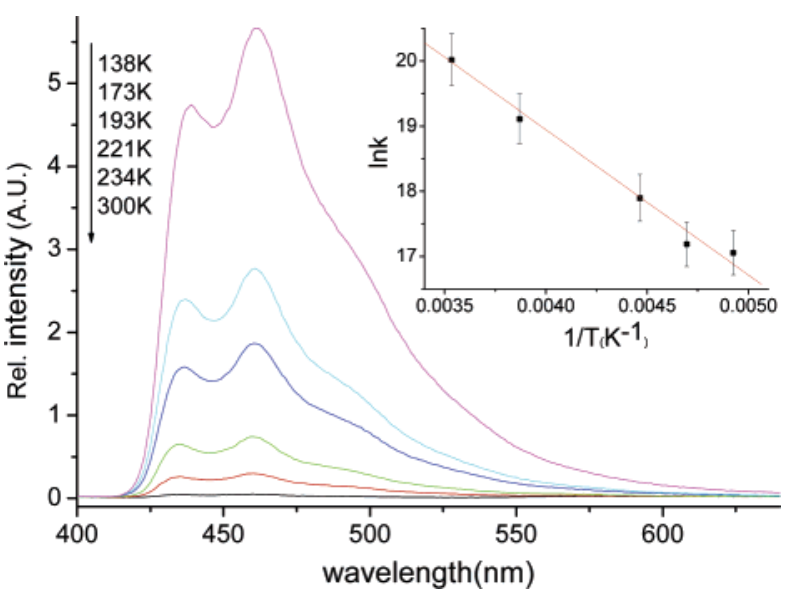

Figure 6. Temperature-dependent emission spectra of 3d in methylcyclohexane. Insert: linear graphical plot of $\ln k$ versus $1 / T$, where $k=$ $\ln \left\{\left(1 / \Phi_{\mathrm{p}}-1-k_{\mathrm{nr}} / k_{\mathrm{r}}\right) k_{\mathrm{r}}\right\}$; see text for the definition.

state pattern, increasing significantly from $1.94 \mathrm{~ns}$ at $298 \mathrm{~K}$ to as long as $890 \mathrm{~ns}$ at $138 \mathrm{~K}$. Assuming a temperatureindependent radiative decay rate $\left(k_{\mathrm{r}}\right)$ for the phosphorescence, the observed temperature-dependent decay rate $k_{\text {obs }}$ can be expressed as $k_{\mathrm{obs}}=k_{\mathrm{r}}+k_{\mathrm{nr}}+k_{\mathrm{nr}}(T)$, in which $k_{\mathrm{nr}}(T)$ represents the temperature-dependent nonradiative deactivation rate and $k_{\mathrm{nr}}$ denotes the temperature-independent radiationless decay rate constant, possibly involving intersystem crossing, etc. Accordingly, the measured quantum yield is deduced to be

$$
\Phi_{\mathrm{p}}=\frac{k_{\mathrm{r}}}{k_{\mathrm{r}}+k_{\mathrm{nr}}+k_{\mathrm{nr}}(T)}
$$

The temperature-dependent radiationless decay rate constant $k_{\mathrm{nr}}(T)$ can be further expressed as an Arrhenius type of thermally deactivated pathway of $k_{\mathrm{nr}}(T)=A \mathrm{e}^{-E_{\mathrm{a}} / R T}$, which is then substituted into eq 3 to obtain eq 4 :

$$
\ln \left\{\left(\frac{1}{\Phi_{\mathrm{p}}}-1-\frac{k_{\mathrm{nr}}}{k_{\mathrm{r}}}\right) k_{\mathrm{r}}\right\}=\ln A-\frac{E_{\mathrm{a}}}{R T}
$$

As indicated by steady-state and time-resolved measurements, the lifetime and intensity of the emission at $<140 \mathrm{~K}$ are nearly temperature independent. Thus $k_{\mathrm{nr}}(T)$ is assumed to be negligible, and $\Phi_{\mathrm{p}}$ expressed in eq 3 can be simplified to $k_{\mathrm{r}} /\left(k_{\mathrm{nr}}+k_{\mathrm{r}}\right)$, which was measured to be $\sim 0.46$ at $138 \mathrm{~K}$. On the other hand, $k_{\text {obs }}$ was resolved to be $1.12 \times 10^{6} \mathrm{~s}^{-1}$ at $138 \mathrm{~K}$. Accordingly $k_{r}$ and $k_{\mathrm{nr}}$ were deduced to be $5.16 \times$ $10^{5}$ and $6.07 \times 10^{5} \mathrm{~s}^{-1}$, respectively. With all parameters provided, the plot of $\ln \left\{\left(1 / \Phi_{\mathrm{p}}-1-k_{\mathrm{nr}} / k_{\mathrm{r}}\right) k_{\mathrm{r}}\right\}$ as a function of the reciprocal of the temperature reveals a straight line, and $E_{\mathrm{a}}$ was deduced to be $4.43 \mathrm{kcal} / \mathrm{mol}$ with a frequency factor $A$ of $1.25 \times 10^{12} \mathrm{~s}^{-1}$ (see insert of Figure 6). The results provide a quantitative estimate of the energy difference between ground vibrational level $(v=0)$ of $\mathrm{T}_{1}$ and a specific channel (or state) that intitates the main deactivation process. Discussion regarding a plausible deactivation pathway will be elaborated in the next section, on theoretical approaches.

\section{Theoretical Approaches}

To further rationalize the photophysical properties of complexes 1-3, ab initio approaches (DFT; see Experimental Section) on the corresponding molecular orbitals involved in the transitions were carried out. The results, according to the chromophores contributing to the frontier orbitals (vide infra), show similar patterns for all complexes $\mathbf{1}-\mathbf{3}$. To avoid redundancy, only the results of $\mathbf{3 d}$ are elaborated here, due to its easily accessible temperature-dependent spectroscopic/ dynamic data (vide supra). The features of the frontier orbitals of $\mathbf{3 d}$ mainly involved in the electronic transitions are depicted in Figure 7, while the descriptions and the energy gap of each transition are listed in Table 5. Apparently, the electron densities of the singlet and triplet states of complex $3 \mathbf{d}$ for the HOMO, HOMO-1, HOMO-2, and HOMO -3 are mainly based on the triazolate, fluorinesubstituted phenyl moieties and Ir atom, while those of the LUMO, on the contrary, are almost completely distributed on the pyridyl moiety, although there are also minor contributions from nonbonding d orbitals of Ir metal. The lowest singlet absorption at $406 \mathrm{~nm}$ (transition 1, HOMO $\rightarrow$ LUMO) corresponds to a transfer of electron density from 4-fluorophenyl to pyridine (ligand to ligand charge transfer, LLCT) and Ir metal to pyridine (MLCT) in character.

At $\sim 355 \mathrm{~nm}$, there is another electronic transition with high oscillator strength, the major contributions of which are ascribed to HOMO -2 and $\mathrm{HOMO}-1 \rightarrow \mathrm{LUMO}$, and the charge density is similar to that calculated for the $406 \mathrm{~nm}$ transition, except for an additional contribution from $\mathrm{C}_{3} \mathrm{~F}_{7}$ substituted triazolate. Another highly allowed transition is located at $\sim 329 \mathrm{~nm}$, which mainly incorporates HOMO-3 $\rightarrow$ LUMO and HOMO-4 $\rightarrow$ LUMO, corresponding to transfer of electron density mainly from Ir metal to pyridine or pyrazole and dimethyl-substituted fmpz or 4-fluorophenyl to pyridine and pyrazole of another ligand. Qualitatively, most of the allowed transition peaks for complex 3d, neglecting the contribution from vibronic transition (i.e. the Franck-Condon envelope), are in good agreement with the observed absorption spectral profiles, supporting the validity of this approach.

For all complexes studied, in contrast to the pure HOMO $\rightarrow$ LUMO as the lowest singlet transition, frontier orbitals associated with the lowest triplet state are rather complicated, involving $\mathrm{HOMO}, \mathrm{HOMO}-1, \mathrm{HOMO}-2$, and $\mathrm{HOMO}-5$ to LUMO (see Table 5 for the case of complex 3d). Similar results were obtained for complexes $\mathbf{1 - 3}$, but they are somewhat different from those of $\operatorname{Ir}(\mathrm{ppy})_{3}$ and its derivatives, which could be reasonably attributed to a ${ }^{3} \pi \pi^{*}$ manifold, mixed to a great extent with the ${ }^{3} \mathrm{MLCT}$ character. ${ }^{32}$ For complexes 1-3, it is apparent, however, that electron densities of the resulting $\mathrm{S}_{0} \rightarrow \mathrm{T}_{1}$ transition state are completely located at the pyridyl moiety, the electron densities of which are contributed from pyrazolate (or triazolate in the anionic $\mathrm{N}^{\wedge} \mathrm{N}$ ligand), phenyl moieties, and the Ir atom $\left(\mathrm{d}_{\pi}\right.$ orbitals). On the other hand, the pyrazole in cyclometalated $\mathrm{C}^{\wedge} \mathrm{N}$ ligand of $\mathbf{1 a}-\mathbf{d}$ has little contribution

(32) Hay, P. J. J. Phys. Chem. A 2002, 106, 1634. 


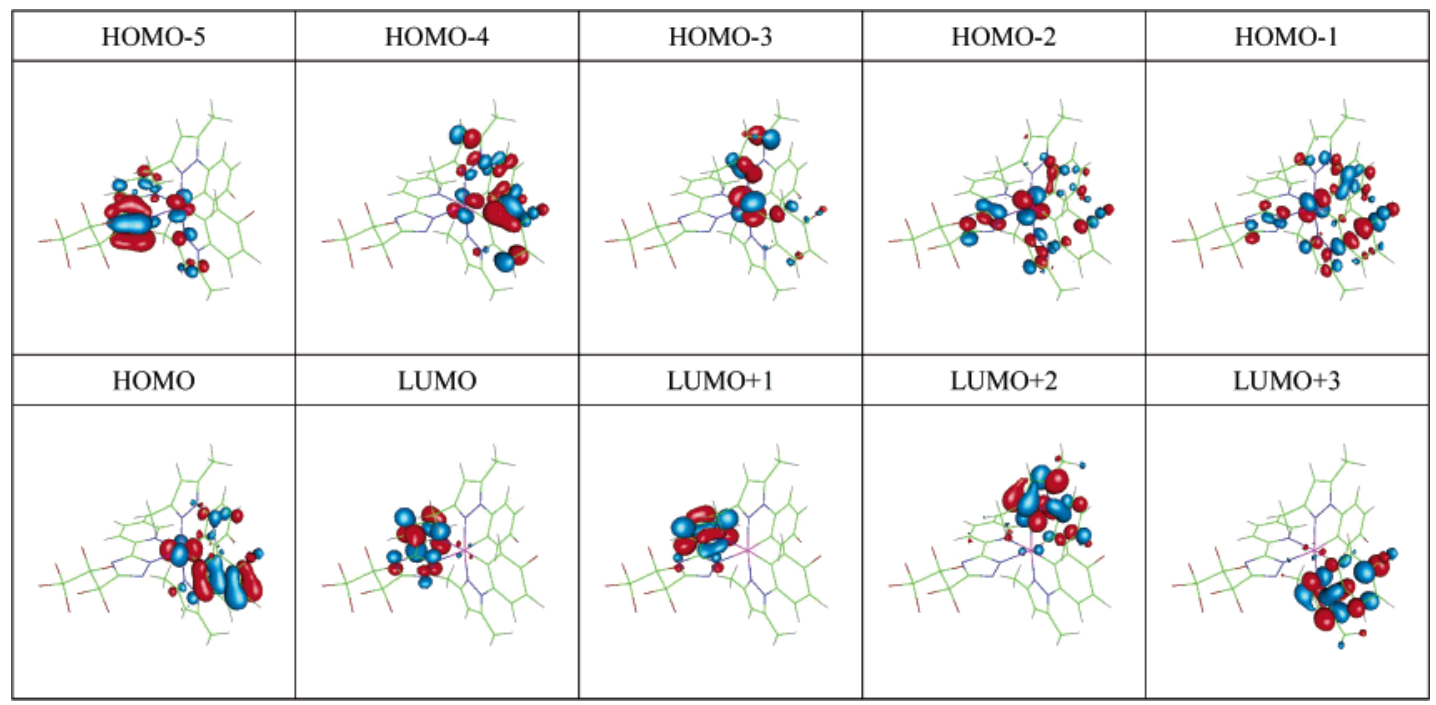

Figure 7. Selected frontier orbitals of complex $\mathbf{3 d}$.

Table 5. Calculated Energy Levels of the Lower Lying Transitions of Complex 3d

\begin{tabular}{|c|c|c|c|c|}
\hline state & assgnt & $E(\mathrm{eV})$ & $\lambda(\mathrm{nm})$ & $(f)$ \\
\hline $\mathrm{S}_{1}$ & HOMO $\rightarrow$ LUMO $(+98 \%)$ & 3.05 & 406.6 & 0.0002 \\
\hline $\mathrm{S}_{2}$ & HOMO $-2 \rightarrow$ LUMO $(+50 \%) ;$ HOMO $-1 \rightarrow$ LUMO $(41 \%)$ & 3.49 & 355.4 & 0.0281 \\
\hline $\mathrm{S}_{3}$ & $\mathrm{HOMO} \rightarrow \mathrm{LUMO}+1(+86 \%) ; \mathrm{HOMO} \rightarrow \mathrm{LUMO}+2(+5 \%)$ & 3.62 & 342.1 & 0.0109 \\
\hline $\mathrm{S}_{4}$ & HOMO $-1 \rightarrow$ LUMO $(+53 \%) ;$ HOMO $-2 \rightarrow$ LUMO $(+38 \%)$ & 3.67 & 337.9 & 0.0018 \\
\hline $\mathrm{S}_{5}$ & $\begin{array}{l}\mathrm{HOMO} \rightarrow \text { LUMO }+2(+79 \%) ; \mathrm{HOMO} \rightarrow \text { LUMO }+1(7 \%) \\
\quad \mathrm{HOMO}-3 \rightarrow \text { LUMO }(6 \%)\end{array}$ & 3.75 & 331.0 & 0.0222 \\
\hline $\mathrm{S}_{6}$ & $\begin{aligned} \mathrm{HOMO}-3 & \rightarrow \mathrm{LUMO}(+70 \%) ; \mathrm{HOMO}-4 \rightarrow \mathrm{LUMO}(+11 \%) ; \\
\mathrm{HOMO} & \rightarrow \mathrm{LUMO}+2(+5 \%)\end{aligned}$ & 3.76 & 329.5 & 0.0453 \\
\hline $\mathrm{S}_{7}$ & $\mathrm{HOMO} \rightarrow \mathrm{LUMO}+3(+84 \%)$ & 3.80 & 326.2 & 0.0302 \\
\hline $\mathrm{S}_{8}$ & HOMO $-4 \rightarrow$ LUMO $(+81 \%) ;$ HOMO $-3 \rightarrow$ LUMO $(12 \%)$ & 3.81 & 325.8 & 0.0006 \\
\hline $\mathrm{S}_{9}$ & $\mathrm{HOMO}-2 \rightarrow \mathrm{LUMO}+1(+44 \%) ; \mathrm{HOMO}-1 \rightarrow \mathrm{LUMO}+1(44 \%)$ & 3.93 & 315.3 & 0.0123 \\
\hline$S_{10}$ & $\begin{array}{l}\mathrm{HOMO}-5 \rightarrow \text { LUMO }(+44 \%) ; \mathrm{HOMO}-2 \rightarrow \mathrm{LUMO}+2(+23 \%) \\
\mathrm{HOMO}-1 \rightarrow \mathrm{LUMO}+2(11 \%) ; \mathrm{HOMO}-3 \rightarrow \mathrm{LUMO}+1(+5 \%)\end{array}$ & 3.95 & 313.5 & 0.0388 \\
\hline $\mathrm{T}_{1}$ & $\begin{array}{c}\text { Triplet Excited States } \\
\text { HOMO } \rightarrow \text { LUMO }(+49 \%) ; \text { HOMO }-1 \rightarrow \text { LUMO }(+15 \%) ;\end{array}$ & 2.98 & 415.9 & $\sim 0$ \\
\hline
\end{tabular}

to the $\mathrm{S}_{0} \rightarrow \mathrm{T}_{1}$ transition, rationalizing the lack of substituent effect on the $N$-phenylpyrazole chromophore (i.e. $\mathrm{C}^{\wedge} \mathrm{N}$ ligand). However, for the triazolate complexes $\mathbf{3 a}-\mathbf{d}$, addition of methyl groups has slightly red shifted the emission by $3-12 \mathrm{~nm}$ in both fluid and solid states (Table 4 ). We believe that the Ir metal center in $\mathbf{3 a}-\mathbf{d}$ is relatively more electron deficient than that of $\mathbf{1 a - d}$ due to the better $\pi$-accepting effect of the triazolate vs that of the pyrazolate $\mathrm{N}^{\wedge} \mathrm{N}$ ligand. This electron deficiency then makes the electron donation ability of the methyl substituents on the $\mathrm{N}$ phenylpyrazole $\mathrm{C}^{\wedge} \mathrm{N}$ chromophores more obvious by raising the $\mathrm{d}_{\pi}$ energy at the $\mathrm{Ir}$ metal center. Moreover, because HOMO, HOMO-1, HOMO-2, and HOMO-5 orbitals that are mainly involved in $\mathrm{S}_{0} \rightarrow \mathrm{T}_{1}$ transition consist of the frontier orbitals among pyrazolate (or triazolate in anionic $\mathrm{N}^{\wedge} \mathrm{N}$ ligand), phenyl moieties, and the Ir atom, substitution on each single moiety is diluted and should cause little effect on the associated photophysical properties, rationalizing the experimental results.

As for the radiationless deactivation pathways, it is noteworthy that because there is no involvement of the $\mathrm{d}_{\pi} *$ orbital up to LUMO +5 in complexes $\mathbf{1 - 3}$, a popularly adopted deactivation mechanism incorporating metal-centered dd transition, which normally results in weakening of the metal-ligand interaction due to its antibonding character, has been discarded..$^{33}$ Alternatively, population at the $T_{1}$ state causes the shift of the electron density from the metal center, phenyl, and pyrazole of the $\mathrm{C}^{\wedge} \mathrm{N}$ ligands to the pyridyl moiety of the unique $\mathrm{N}^{\wedge} \mathrm{N}$ ligand, resulting in the weakening of all $\mathrm{Ir}$-ligand bonding interactions. This, in combination with $\mathrm{T}_{1}$ being in a high-energy blue region, may lead to a shallow potential energy surface (PES) in the $\mathrm{T}_{1}$ state; thus, as for an extreme case, a surface crossing of PES between $S_{0}$ and $\mathrm{T}_{1}$ is possible. Thus, upon excitation, the $\mathrm{T}_{1}$ state is populated right after fast $\mathrm{S}_{1} \rightarrow \mathrm{T}_{n}$ ISC, followed by the internal conversion and vibrational relaxation (i.e. solvent deactivation). Subsequently, radiationless deactivations are executed via thermal activation to certain vibrational levels close to the section of the $T_{1}-S_{0}$ surface through the facile metalligand bond stretching discussed above. Particularly, the contribution of the fluorophenyl $\rightarrow$ pyridyl transition to the $\mathrm{T}_{1}$ state is $>40 \%$ for complexes $\mathbf{1 - 3}$ (see Supporting Information). The resulting transition should weaken the corresponding Ir-phenyl (or Ir-pyridyl) bonds, which, in a sense, may trigger the deactivation processs. Although detailed insights into the deactivation mechanism are still

(33) (a) Manuta, D. M.; Lees, A. J. Inorg. Chem. 1986, 25, 1354. (b) Demas, J. N.; DeGraff, B. A. Anal. Chem. 1991, 63, 829A. 
pending for resolution, the experimentally extracted activation energy of $4.43 \mathrm{kcal} / \mathrm{mol}$ provides a quantitative estimate of the energy difference between the ground vibrational level of $\mathrm{T}_{1}$ and the surface crossing section, while the preexponential factor of $1.25 \times 10^{12} \mathrm{~s}^{-1}$ reflects the vibrational frequency of the weakly bonding modes channeling into the major radiationless pathway.

\section{Conclusion}

In conclusion, designing a highly efficient blue phosphorescence emitter based on a third-row transition metal is a demanding task. Achieving the phosphorescence toward the blue hue requires pushing either $\pi \pi^{*}$ or MLCT to a higher energy gap, which normally calls for ligands with an electron-withdrawing substituent or with less extended conjugation. The incorporation of an electron-withdrawing $\mathrm{F}$ atom and/or $\mathrm{CF}_{3}$ group at the electron-rich segment of a chelate ligand is expected to increase the ligand-centered $\pi \pi^{*}$ energy gap and simultaneously destabilized the MLCT transition. On the other hand, the less extended conjugation would make the localization of the excessive energy to specific bonds, i.e., the metal-ligand dative interactive, increasingly significant. Likewise, due to the increase of energy gaps, frontier orbitals contributing to the higher lying HOMOs may spread out to any ligand fragments possessing $\pi$ chromophores, resulting in a mixing of various types (MLCT, ILCT, LLCT) of transitions in the $\mathrm{T}_{1}$ state. The net results caused the weakening of all corresponding metalligand bonds. Both factors are believed to induce radiationless deactivation, as demonstrated in complexes $\mathbf{1 - 3}$. While the former is an intrinsic demand in choosing a ligand with $\pi$ chromophores of interest, the latter may be overcome by introducing the strong field auxiliary ligands or the non- $\pi$ electron conjugated ligands so that the LLCT can be reduced significantly. Finally, bright blue phosphorescent emission with quantum efficiency $\Phi_{\mathrm{p}} \sim 0.13$ was observed for complex 1a in the solid state at room temperature. Our results point to the prospect of using this complex as an emitter for blue or even white phosphorescent OLED devices. Focus on this issue is currently in progress.

Acknowledgment. This work was funded by the National Science Council of Taiwan, ROC, under Grants NSC 932113-M-007-012 and NSC 93-2752-M-002-002-PAE. We are also grateful to the National Center for High-Performance Computing of Taiwan for generous amounts of computing time.

Supporting Information Available: The calculated energy levels and the associated frontier orbitals of the DFT calculations on complexes $\mathbf{1 - 3}$ and X-ray crystallographic data files (CIF) for complexes $1 \mathbf{a}$ and $\mathbf{3 d}$. This material is available free of charge via the Internet at http://pubs.acs.org.

IC050311G 